\title{
Heavy Vector and Axial-Vector Mesons in Hot and Dense Asymmetric Strange Hadronic Matter
}

\author{
Arvind Kumar* and Rahul Chhabrat \\ Department of Physics, Dr. $B R$ Ambedkar National Institute \\ of Technology Jalandhar, Jalandhar - 144011,Punjab, India
}

\begin{abstract}
We calculate the effects of finite density and temperature of isospin asymmetric strange hadronic matter, for different strangeness fractions, on the in-medium properties of vector $\left(D^{*}, D_{s}^{*}, B^{*}, B_{s}^{*}\right)$ and axial-vector $\left(D_{1}, D_{1 s}, B_{1}, B_{1 s}\right)$ mesons, using chiral hadronic $\mathrm{SU}(3)$ model and QCD sum rules. We focus on the evaluation of in-medium mass-shift and shift in decay constant of above vector and axial-vector mesons. In QCD sum rule approach, the properties, e.g., the masses and decay constants of vector and axial-vector mesons are written in terms of quark and gluon condensates. These quark and gluon condensates are evaluated in the present work within chiral SU(3) model, through the medium modification of, scalar-isoscalar fields $\sigma$ and $\zeta$, the scalar-isovector field $\delta$ and scalar dilaton field $\chi$, in the strange hadronic medium which includes both nucleons as well as hyperons. As we shall see in detail, the masses and decay constants of heavy vector and axialvector mesons are affected significantly due to isospin asymmetry and strangeness fraction of the medium and these modifications may influence the experimental observables produced in heavy ion collision experiments. The results of present investigations of in-medium properties of vector and axial-vector mesons at finite density and temperature of strange hadronic medium may be helpful for understanding the experimental data from heavy-ion collision experiments in-particular for the Compressed Baryonic Matter (CBM) experiment of FAIR facility at GSI, Germany.
\end{abstract}

Keywords: Dense hadronic matter, strangeness fraction, heavy-ion collisions, effective chiral model, QCD sum rules, heavy mesons.

PACS numbers : -14.40.Lb ,-14.40.Nd,13.75.Lb

${ }^{*}$ Electronic address: iitd.arvind@gmail.com, kumara@nitj.ac.in

$\dagger$ Electronic address: rahulchhabra@ymail.com, 


\section{INTRODUCTION}

The aim of relativistic heavy-ion collision experiments is to explore the different phases of QCD phase diagram so as to understand the underlying strong interaction physics of Quantum Chromodynamics. The different regions of QCD phase diagram can be explored by varying the beam energy in the high energy heavy-ion collision experiments. The nucleusnucleus collisions at the Relativistic Heavy Ion Collider (RHIC) and Large Hadron Collider (LHC) experiments explore the region of the QCD phase diagram at low baryonic densities and high temperatures. However, the objective of the Compressed Baryonic Matter (CBM) experiment of FAIR project (at GSI Germany) is to study the region of phase diagram at high baryonic density and moderate temperature. In nature these kind of phases may exist in astrophysical compact objects, e.g., in neutron stars. Among the many different observables which may be produced in CBM experiment, the one of them may be the production of mesons having charm quark or antiquark. Experimentally, charm meson spectroscopy as well as their in-medium properties are also of interest from the point of view of PANDA experiment of FAIR project, where $\bar{p} A$ collisions will be performed. The possibility of the production of open or hidden charm mesons motivate the theoretical physicist to study the properties of these mesons in dense nuclear matter. The discovery of many open or hidden charm or bottom mesons at CLEO, Belle or BABAR experiments [1 3] attract the attentions of theoretical groups to study the properties of these mesons.

In the present paper, our objective is to work out the in-medium masses and decay constants of heavy charmed vector $\left(D^{*}, D_{s}^{*}\right)$ and axial-vector $\left(D_{1}, D_{1 s}\right)$ as well as bottom vector $\left(B^{*}, B_{s}^{*}\right)$ and axial-vector $\left(B_{1}, B_{1 s}\right)$ mesons in the isospin asymmetric strange hadronic medium, at finite density and temperature The initial interest in the understanding of the properties of heavy flavor mesons was stimulated from the observation of $J / \psi$ suppression phenomenon [4-7]. In the high temperature regime, the $J / \psi$ suppression can be explained either using the color screening or statistical hadronization model [8 12]. However, for the moderate temperature and finite baryonic densities the effects of hadronic absorptions and

comover interactions also come into picture and cannot be neglected [13]. The evaluation of in-medium properties of open charm $D$ mesons may also play important role in understanding of $J / \psi$ suppression in heavy-ion collisions. The reason is, the higher charmonium states are considered as major source of $J / \psi$ mesons. However, if the $D$ mesons undergo mass 
drop in the nuclear matter and the in-medium mass of $D \bar{D}$ pairs falls below the threshold value of excited charmonium states, then these charmonium states can also decay to $D \bar{D}$ pairs and may cause a decrease in the yield of $J / \psi$ mesons. The in-medium properties of $D$ and $\bar{D}$ mesons may also play an important role in revealing the possibility of formation of charmed mesic nuclei. The charmed mesic nuclei are the bound states of charm mesons and nucleon formed through strong interactions. In Ref. [14], the mean field potentials of $D$ and $\bar{D}$ mesons were calculated using the quark meson coupling (QMC) model under local density approximation and the possibilities of the formation of bound states of $D^{-}, D^{0}$ and $\bar{D}^{0}$ mesons with $\mathrm{Pb}(208)$ were examined. The properties of charmed mesons in the nuclei had also been studied using the unitary meson-baryon coupled channel approach and incorporating the heavy-quark spin symmetry [15, 16]. The theoretical investigations of open or hidden charmed meson properties at finite density and temperature of the nuclear matter may help us in understanding their production rates, decay constants, decay widths, etc., in heavy-ion collision experiments.

The in-medium properties of open charm and bottom mesons are evaluated using the quark meson coupling model in Refs. [14, 17]. The medium modified masses of $D$ mesons, calculated within QMC model, were used as input for the study of mass modification of $J / \psi$ mesons in the nuclear matter [18]. Borel transformed QCD sum rules, involving operator product expansion (OPE) upto dimension-4, were used to investigate the in-medium modifications of pseudoscalar $D$ mesons and the impact of these modifications on charmonium suppression in Ref. [19]. The study of open charm mesons within QCD sum rules were further carried out in Ref. [20], where even as well as odd part of OPE was used to study mass-spitting between pseudoscalar $D-\bar{D}$ doublet. In the study of mass shift of $D$ mesons in Ref. [20], a positive mass shift of about $+45 \mathrm{MeV}$ was obtained at normal nuclear saturation density, whereas in the work of Ref. [19], a negative mass-shift of magnitude $50 \mathrm{MeV}$ was observed. The properties of pseudoscalar $B$ mesons as well as charm strange $D_{s}$ mesons were also studied in Ref. [20] and these studies were further extended to the scalar $D$ mesons in Ref. [21]. In Ref. [22], the OPE for pseudoscalars $D$ and $B$ mesons was performed for both, the normal-order operators and also for non-normal-ordered operators. A projection method to calculate the higher order contributions to OPE, for in-medium QCD sum rules of $D$ mesons was used in Ref. [23]. A study on the decomposition of higher dimensional condensates into vacuum part and a medium part was done recently in [24]. The heavy 
quark expansion for the study of $D$ and $B$ mesons in the nuclear matter was performed in [25]. The Wilson coefficients and four quark condensates in the QCD sum rules for the medium modification of $D$ mesons were calculated in Ref. [26].

The properties of open and hidden charm mesons have also been studied to great extent in coupled channel approach [27-35]. Using the separable potential technique between mesonbaryon interactions and $\mathrm{SU}(3)$ symmetry among the $u, d$ and $c$ quarks, the spectral properties of $D$ mesons were investigated in the nuclear matter at zero [27] and finite temperatures [28]. In the work of references [29, 30], the properties of $D$ mesons were studied in $\mathrm{SU}(4)$ sector including the strangeness channel also and breaking SU(4) symmetry through the exchange of vector mesons. In Ref. [31], the properties of $D$ and $\bar{D}$ mesons were investigated selfconsistently in coupled channel approach including the Pauli blocking effects and mean field potential of baryons. The properties of scalar charm resonances and hidden charm resonances were also studied using coupled channel method in [32]. The study of open charm mesons within coupled channel approach was further improved by using heavy quark spin-flavour symmetry and the in-medium properties of charmed pseudoscalar and vector mesons as well as charm baryon resonances $\Lambda_{c}, \Sigma_{c}, \Xi_{c}$ and $\Omega_{c}$ were investigated [16, 33] . In Ref. [34], the chiral unitary coupled channel approach was used to study the interactions of light vector mesons with baryon and nuclei and the possibilities of the formation of quasibound states of vector mesons with baryons were observed. These studies were also extended to charm sector where hidden charm states were observed and which has consequences on $J / \psi$ suppression [34]. The baryons states with charm degree of freedom can be generated dynamically within the coupled channel approach and also incorporating the heavy quark spin symmetry [35].

The chiral hadronic SU(3) model is generalized to SU(4) and SU(5) sector, for investigating the in-medium properties of pseudoscalar $D$ and $B$ mesons [36 38]. The in-medium mass modifications of scalar, vector and axial-vector heavy $D$ and $B$ mesons were investigated using the QCD sum rules in the nuclear matter in [39, 40]. In Ref. [41], we studied the mass-modifications of scalar, vector and axial vector heavy charmed and bottom mesons at finite density of the nuclear matter using the chiral SU(3) model and QCD sum rules. In this approach, we evaluated the medium modifications of quark and gluon condensates in the nuclear matter through the medium modification of scalar isoscalar fields $\sigma$ and $\zeta$ and the scalar dilaton field $\chi$. In the present paper, we shall evaluate the properties of vector and axial-vector mesons in the hot and dense isospin asymmetric strange hadronic medium. 
In the QCD sum rules, the properties of above mesons are modified through the quark and gluon condensates. Within the chiral hadronic model the quark and gluon condensates are written in terms of scalar fields $\sigma, \zeta$ and $\delta$ and the scalar dilaton field $\chi$. We shall evaluate the $\sigma, \zeta, \delta$ and $\chi$ fields and hence the quark and gluon condensates in the medium consisting of nucleons and hyperons and shall find the mass shift and shift in decay constants of heavy vector and axial vector mesons.

The study of decay constant of heavy mesons play important role in understanding the strong decay of heavy mesons, their electromagnetic structure as well radiative decay width.

The study of B meson decay constants is important for $B_{d}-\bar{B}_{d}$ and $B_{s}-\bar{B}_{s}$ mixing [42]. The decay constants of vector $D^{*}$ and $B^{*}$ mesons are helpful for calculations of strong coupling in $D^{*} D \pi$ and $B^{*} B \pi$ mesons using light cone sum rules [43]. An extensive literature is available on the calculations of decay constants of heavy mesons in the free space, for example, the QCD sum rules based on OPE of two-point correlation function, heavy-quark expansion [44], sum rules in heavy-quark effective theory [45, 46] and sum rules with gluon radiative corrections to the correlation functions upto two loop [47-49] or three loop [50]. However, the in-medium modifications of decay constants of heavy mesons had been studied in symmetric nuclear matter only [51, 52]. The thermal modification (at zero baryonic density) of decay constants of heavy vector mesons was investigated in Ref. [53] and it was observed that the values of decay constants remained almost constant upto $100 \mathrm{MeV}$, but above this decrease sharply with increase in temperature.

We shall present this work as follows: In Sec. II, we shall briefly describe the chiral SU(3) model which is used to evaluate the quark and gluon condensates in the strange hadronic matter. Section III will introduce the QCD sum rules which we shall use in the present work along with chiral model to evaluate the in-medium properties of mesons. In Sec. IV, we shall present our results of present investigation and possible discussion on these results. Section V will summarize the present work.

\section{CHIRAL SU(3) MODEL}

The basic theory of strong interaction, the QCD, is not directly applicable in the nonperturbative regime. To overcome this limitation, the effective theories, constrained by the basic properties, e.g., chiral symmetry and scale invariance of QCD, are constructed. 
The chiral SU(3) model is one such effective model based on the non-linear realization and broken scale invariance as well as spontaneous breaking properties of chiral symmetry [54, 55]. The glueball field $\chi$ is introduced in the model to account for the broken scale invariance properties of QCD. The model had been used successfully in the literature to study the properties of hadrons at finite density and temperature of the nuclear and strange hadronic medium. The general Lagrangian density of the chiral SU(3) model involve the kinetic energy terms, the baryon meson interactions, self interaction of vector mesons, scalar mesons-meson interactions as well as the explicit chiral symmetry breaking term [54]. From the Lagrangian densities of the chiral SU(3) model, using the mean field approximation, we find the coupled equations of motion for the scalar fields $\sigma, \zeta, \delta$ and the scalar dilaton field $\chi$ [55]. These coupled equations of motion for the above fields are solved for the different values of isospin asymmetry parameter $I$, and strangeness fraction $f_{s}$, of the hadronic medium at finite density and temperature. The isospin asymmetry parameter, $I$, is defined by the relation, $I=\frac{\rho_{n}-\rho_{p}}{2 \rho_{B}}$, where, $\rho_{n}$ and $\rho_{p}$, are the number densities of neutrons and protons respectively and $\rho_{B}$ is baryon density. The strangeness fraction of the medium is given by, $f_{s}=\frac{\sum_{i}\left|s_{i}\right| \rho_{i}}{\rho_{\bar{B}}}$. Here, $s_{i}$, is the number of strange quarks and $\rho_{i}$ is the number density of $i^{\text {th }}$ baryon [55].

In the present work, for the evaluation of vector and axial-vector meson properties using QCD sum rules, we shall need the light quark condensates $\langle\bar{u} u\rangle$ and $\langle\bar{d} d\rangle$, the strange quark condensate $\langle\bar{s} s\rangle$ and the scalar gluon condensate $\left\langle\frac{\alpha_{s}}{\pi} G_{\mu \nu}^{a} G^{\mu \nu a}\right\rangle$. In the chiral effective model, the explicit symmetry breaking term is introduced to eliminate the Goldstone bosons and can be used to extract the scalar quark condensates, $\langle q \bar{q}\rangle$, in terms of scalar fields $\sigma, \zeta, \delta$ and $\chi$. We write [55],

$$
\begin{aligned}
& \sum_{i} m_{i} \bar{q}_{i} q_{i}=-\mathcal{L}_{S B} \\
= & \left(\frac{\chi}{\chi_{0}}\right)^{2}\left(\frac{1}{2} m_{\pi}^{2} f_{\pi}(\sigma+\delta)+\frac{1}{2} m_{\pi}^{2} f_{\pi}(\sigma-\delta)+\left(\sqrt{2} m_{k}^{2} f_{k}-\frac{1}{\sqrt{2}} m_{\pi}^{2} f_{\pi}\right) \zeta\right) .
\end{aligned}
$$

From Eq. (22), light scalar quark condensates $\langle\bar{u} u\rangle$ and $\langle\bar{d} d\rangle$, and the strange quark condensate $\langle\bar{s} s\rangle$, can be written as,

$$
\begin{aligned}
& \langle\bar{u} u\rangle=\frac{1}{m_{u}}\left(\frac{\chi}{\chi_{0}}\right)^{2}\left[\frac{1}{2} m_{\pi}^{2} f_{\pi}(\sigma+\delta)\right], \\
& \langle\bar{d} d\rangle=\frac{1}{m_{d}}\left(\frac{\chi}{\chi_{0}}\right)^{2}\left[\frac{1}{2} m_{\pi}^{2} f_{\pi}(\sigma-\delta)\right],
\end{aligned}
$$


and

$$
\langle\bar{s} s\rangle=\frac{1}{m_{s}}\left(\frac{\chi}{\chi_{0}}\right)^{2}\left[\left(\sqrt{2} m_{k}^{2} f_{k}-\frac{1}{\sqrt{2}} m_{\pi}^{2} f_{\pi}\right) \zeta\right],
$$

respectively. Also, we know that, due to broken scale invariance property of QCD, the trace of energy momentum tensor is non-vanishing (trace anomaly) and is equal to the scalar gluon condensates. The trace anomaly property of QCD can be mimicked in effective chiral model through the scale breaking Lagrangian density which can be further used to evaluate the trace of energy momentum tensor. Comparing, the trace of energy momentum tensor evaluated from effective chiral model, to the trace of energy momentum tensor of basic QCD theory, we can express the gluon condensate in terms of scalar fields $\sigma, \zeta, \delta$ and $\chi$ [54, 55]. This relation is given by,

$$
\left\langle\frac{\alpha_{s}}{\pi} G_{\mu \nu}^{a} G^{a \mu \nu}\right\rangle=\frac{8}{9}\left[(1-d) \chi^{4}+\left(\frac{\chi}{\chi_{0}}\right)^{2}\left(m_{\pi}^{2} f_{\pi} \sigma+\left(\sqrt{2} m_{k}^{2} f_{k}-\frac{1}{\sqrt{2}} m_{\pi}^{2} f_{\pi}\right) \zeta\right)\right] .
$$

Equations (3) to (6) give us the expressions for different scalar quark condensates and gluon condensate in terms of scalar fields within chiral SU(3) model. It should be noted that, for massless quarks, the second term in Eq. (6) , arising from explicit symmetry breaking will be absent and the scalar gluon condensate becomes proportional to the fourth power of the dilaton field $\chi$, in the chiral $\mathrm{SU}(3)$ model.

\section{QCD SUM RULES FOR VECTOR AND AXIAL-VECTOR HEAVY MESONS IN STRANGE HADRONIC MATTER}

In this section, we shall discuss the QCD sum rules [39, 40], to be used later along with the chiral $\mathrm{SU}(3)$ model, for the evaluation of in-medium properties of vector and axialvector mesons in asymmetric strange hadronic matter. We know that, the starting point in the QCD sum rules is to write the two-point correlation function, $\Pi_{\mu \nu}(q)$, which is the Fourier transform of the expectation value of time ordered product of isospin averaged currents $J_{\mu}(x)$ and can be decomposed into vacuum part, a static nucleon part and pion bath contribution [41, 56, 57]. For the vector and axial-vector mesons the average particleantiparticle currents are given by, $J_{\mu}(x)=J_{\mu}^{\dagger}(x)=\frac{\bar{c}(x) \gamma_{\mu} q(x)+\bar{q}(x) \gamma_{\mu} c(x)}{2}$ and $J_{5 \mu}(x)=J_{5 \mu}^{\dagger}(x)=$ $\frac{\bar{c}(x) \gamma_{\mu} \gamma_{5} q(x)+\bar{q}(x) \gamma_{\mu} \gamma_{5} c(x)}{2}$, respectively. Here, $q$, denotes the $u, d$ or $s$ quark (depending upon type of meson under investigation), whereas, $c$, denotes the heavy charm quark (for $B$ mesons the quark, $c$, will be replaced by bottom, $b$, quark). We know that the charmed mesons $D^{+}$, 
$D^{-}, D^{0}$ and $\bar{D}^{0}$ have the quark compositions, $c \bar{d}, d \bar{c}, c \bar{u}$ and $u \bar{c}$, respectively. The mesons $D^{+}$and $D^{-}$are particle-antiparticles to each-other and similarly, $D^{0}$ and $\overline{D^{0}}$ mesons. The above listed charm mesons belong to the isospin, $D\left(D^{+}, D^{0}\right)$ and $\bar{D}\left(D^{-}, \bar{D}^{0}\right)$ doublets. In the present work, we shall find the average mass-shift for particle-antiparticle pairs, i.e., for, $D^{+}$and $D^{-}$, and for, $D^{0}$ and $\overline{D^{0}}$, under centroid approximation [19, 40]. In this way, we will also be able to study the mass-splitting of isospin doublet due to isospin asymmetry of the medium. To find the mass splitting of particles and antiparticles in the nuclear medium one has to consider the even and odd part of QCD sum rules [20]. For example, in Ref. [20] the mass splitting between pseudoscalar $D$ and $\bar{D}$ mesons was investigated using the even and odd QCD sum rules, whereas in [19, 39, 40] the mass-shift of $D$ mesons was investigated under centroid approximation. It means, in the present work we assumed the degeneracy between particle-antiparticle mass. Note that the sum rules for $D^{0}$ can be obtained from the $D^{+}$just by replacing $d$ quark by $u$ quark [22] .

In literature of QCD sum rules, the pion bath contributions are used to evaluate the effects of finite temperature of the medium on the properties of mesons [56, 57]. In our present approach, however, the effects of finite temperature of the medium on the properties of vector and axial-vector mesons are evaluated through the temperature dependence of scalar fields $\sigma, \zeta, \delta$ and $\chi$, which will appear through static one nucleon part [36, 41, 55, 58, 59]. For the case of vector and axial vector mesons, the correlation function $T_{\mu \nu}^{N}(\omega, \boldsymbol{q})$, which appear in the static nucleon part, can be written in terms of forward scattering amplitudes $T_{N}(\omega, \boldsymbol{q})$ (corresponding to vector and axial-vector mesons) and $T_{N}^{0}(\omega, \mathbf{q})$ (corresponding to scalar and pseudoscalar mesons), as follows [51, 60],

$$
T_{\mu \nu}^{N}(\omega, \boldsymbol{q})=T_{N}(\omega, \mathbf{q})\left(\frac{q_{\mu} q_{\nu}}{q^{2}}-g_{\mu \nu}\right)+T_{N}^{0}(\omega, \mathbf{q}) \frac{q_{\mu} q_{\nu}}{q^{2}} .
$$

Note that in the present work, we are interested in the properties of vector and axial-vector mesons and therefore, second term of Eq. (77) will not contribute. The forward scattering amplitude $T_{N}(\omega, \mathbf{q})$, in the limit, $\mathbf{q} \rightarrow 0$, can be written in terms of spin averaged spectral density $\rho$, as follows [52, 60],

$$
T_{N}(\omega, \mathbf{q})=\int_{-\infty}^{+\infty} d u \frac{\rho(u, \mathbf{q}=0)}{u-\omega-i \epsilon}=\int_{0}^{\infty} d u^{2} \frac{\rho(u, \mathbf{q}=0)}{u^{2}-\omega^{2}} .
$$

Near the pole positions of vector and axial vector mesons, the phenomenological spectral densities can be further related to the $D^{*} N$ and $D_{1} N$ scattering matrix $\mathcal{T}$, and can be 
parametrized with three unknown parameters $a, b$ and $c$ as discussed in Refs. [19, 39 41]. Also, the in-medium mass shift, $\delta m_{D^{*} / D_{1}}$, of vector and axial-vector mesons can be related to their scattering length $a_{D^{*} / D_{1}}$, through parameter $a$ and is given by [19, 39] 41],

$$
\begin{aligned}
\delta m_{D^{*} / D_{1}} & =\sqrt{m_{D^{*} / D_{1}}^{2}+\left(\frac{\rho_{B}}{2 m_{N}} \frac{a}{f_{D^{*} / D_{1}}^{2} m_{D^{*} / D_{1}}^{2}}\right)}-m_{D^{*} / D_{1}} \\
& =\sqrt{m_{D^{*} / D_{1}}^{2}+\left(-\frac{\rho_{B}}{2 m_{N}} 8 \pi\left(m_{N}+m_{D^{*} / D_{1}}\right) a_{D^{*} / D_{1}}\right)}-m_{D^{*} / D_{1}} .
\end{aligned}
$$

The shift in the decay constant of vector or axial vector mesons can be written as [51],

$$
\delta f_{D^{*} / D_{1}}=\frac{1}{2 f_{D^{*} / D_{1}} m_{D^{*} / D_{1}}^{2}}\left(\frac{\rho_{B}}{2 m_{N}} b-2 f_{D^{*} / D_{1}}^{2} m_{D^{*} / D_{1}} \delta m_{D^{*} / D_{1}}\right) .
$$

From Eqs. (9) and (10) we observe that, to find the value of mass shift and shift in decay constant of mesons, we first need to know the value of unknown parameters $a$ and b. For achieving this, the equations for Borel transformation of the scattering matrix on the phenomenological side are equated to the Borel transformation of the scattering matrix for the OPE side [19, 39, 40]. The detailed expressions for the Borel transformed equations of heavy vector and axial-vector mesons can be found in Refs. [40, 41]. The unknown parameters $a$ and $b$, will appear in these Borel transformed equations. To obtain the values of these two unknown parameters, the Borel transformed equation of the meson under study is differentiated w.r.t. $\frac{1}{M^{2}}$, so that we could have two equations and two unknowns. By solving those two coupled equations we will be able to get the values of parameters, $a$ and $b$.

Also note that, the Borel transformed equations of vector and axial-vector mesons will depend on the nucleon expectation values of quark condensates $\langle\bar{q} q\rangle,\left\langle q^{\dagger} i D_{0} q\right\rangle,\left\langle\bar{q} g_{s} \sigma G q\right\rangle$ and $\left\langle\bar{q} i D_{0} i D_{0} q\right\rangle$, and the gluon condensate $\left\langle\frac{\alpha_{s}}{\pi} G^{a}{ }_{\mu \nu} G^{a \mu \nu}\right\rangle$ [41]. The nucleon expectation values of the operators can be expressed in terms of the expectation value of operators at finite baryonic density and vacuum expectation value [41, 56, 57, 60]. As discussed earlier, in Sec. II, the scalar quark condensate $\langle\bar{q} q\rangle$ and the gluon condensates $\left\langle\frac{\alpha_{s}}{\pi} G^{a}{ }_{\mu \nu} G^{a \mu \nu}\right\rangle$, can be evaluated within chiral SU(3) model. The condensates, $\left\langle\bar{q} g_{s} \sigma G q\right\rangle$ and $\left\langle\bar{q} i D_{0} i D_{0} q\right\rangle$, can be approximated in terms of scalar quark condensates $\langle\bar{q} q\rangle_{\rho_{B}}[61]$. The value of $\langle\bar{q} q\rangle$, evaluated within chiral SU(3) model, can be used in the equations of $\left\langle\bar{q} g_{s} \sigma G q\right\rangle$ and $\left\langle\bar{q} i D_{0} i D_{0} q\right\rangle$, for an improved evaluation of these condensates within chiral model. The value of quark 
condensate, $\left\langle q^{\dagger} i D_{0} q\right\rangle$, for light quark is equal to $0.18 G e V^{2} \rho_{B}$ and for strange quark it is $0.018 \mathrm{GeV}^{2} \rho_{B}$ [61].

Note that, the bottom mesons, i.e., $B^{+}, B^{-}, B^{0}$ and $\bar{B}^{0}$, have the quark compositions, $u \bar{b}, b \bar{u}, d \bar{b}$ and $b \bar{u}$, respectively. Comparing the quark compositions of charm and bottom mesons, e.g., for $B^{+}$and $\bar{D}^{0}$, we observe that both have same light $u$ quark and differ in heavy flavor antiquark. In the QSR equations of $D$ mesons, the quark condensates corresponding to only light quark appear along with the masses of heavy quark and mesons. It means QSR equation of $B^{+}$will be differ from that of $\bar{D}^{0}$ mere by masses of heavy flavor mesons (both have same light quark). Same correspondence exist for $B^{-}$and $D^{0}, B^{0}$ and $D^{-}$and $\bar{B}^{0}$ and $D^{+}$. Thus sum rules for the bottom $B$ mesons can be obtained from the sum rules of $D$ mesons by replacing, $m_{c}$ by $m_{b}, D^{*}\left(D_{1}\right)$ by $B^{*}\left(B_{1}\right)$ and also by replacing $\Lambda_{c}\left(\Sigma_{c}\right)$ by $\Lambda_{b}\left(\Sigma_{b}\right)$. The same strategy was applied to obtain the OPE for pseudoscalar $B$ mesons from $D$ mesons in Refs. [22, 39, 40, 51, 52]. When we move from non-strange to strange mesons, the light quark is replaced by strange quark. Therefore, the QCD sum rules for the strange vector mesons are obtained by using the strange condensates in place of light quark condensates. Hilger in Ref. [20] used the same strategy for obtaining the sum rules for pseudoscalar $D_{s}$ mesons from $D$ meson sum rules. Now the sum rule equations of $D_{s}$ mesons will also be differ from $B_{s}$ mesons by the mass of heavy flavor mesons only and therefore, sum rules for strange $B_{s}$ mesons are obtained by replacing masses of $D_{s}$ mesons by corresponding masses of $B_{s}$ mesons.

\section{RESULTS AND DISCUSSIONS}

In this section, we shall discuss the results of present investigation of in-medium mass shift and shift in decay constant of vector $\left[D^{*}\left(D^{*+}, D^{* 0}, D_{s}^{*}\right)\right.$ and $\left.B^{*}\left(B^{*+}, B^{* 0}, B_{s}^{*}\right)\right]$ and axial-vector $\left[D_{1}\left(D_{1}^{+}, D_{1}^{0}, D_{1 s}\right)\right.$ and $\left.B_{1}\left(B_{1}^{+}, B_{1}^{0}, B_{1 s}\right)\right]$ mesons in isospin asymmetric strange hadronic matter. First we list the values of various parameters used in the present work on vector and axial-vector mesons. Nuclear matter saturation density adopted in the present investigation is $0.15 \mathrm{fm}^{-3}$. The coupling constants of heavy baryons with nucleons and heavy charmed/bottom mesons appear in the hadronic matrix elements of the heavy baryon transitions to nucleon. These coupling constants are calculated using the light cone QCD

sum rules [62]. The average value of coupling constants, $g_{D^{*} N \Lambda_{c}}$ and $g_{D^{*} N \Sigma_{c}}$ is 3.86 [40, 51, 


\begin{tabular}{|c|c|c|c|c|c|c|}
\hline & $D^{*+}$ & $D^{* 0}$ & $B^{* 0}$ & $B^{*+}$ & $D_{s}^{*}$ & $B_{s}^{*}$ \\
\hline$m(\mathrm{GeV})$ & 2.01 & 2.006 & 5.325 & 5.323 & 2.112 & 5.415 \\
\hline$f(\mathrm{GeV})$ & 0.270 & 0.270 & 0.195 & 0.195 & $1.16 f_{D^{*}}$ & $1.16 f_{B^{*}}$ \\
\hline$s_{0}\left(\mathrm{GeV}^{2}\right)$ & 6.5 & 6.5 & 35 & 35 & 7.5 & 38 \\
\hline \hline & $D_{1}^{+}$ & $D_{1}^{0}$ & $B_{1}^{0}$ & $B_{1}^{+}$ & $D_{1 s}$ & $B_{1 s}$ \\
\hline$m(\mathrm{GeV})$ & 2.423 & 2.421 & 5.723 & 5.721 & 2.459 & 5.828 \\
\hline$f(\mathrm{GeV})$ & 0.305 & 0.305 & 0.255 & 0.255 & $1.16 f_{D_{1}}$ & $1.16 f_{B_{1}}$ \\
\hline$s_{0}\left(\mathrm{GeV}^{2}\right)$ & 8.5 & 8.5 & 39 & 39 & 9.5 & 41 \\
\hline
\end{tabular}

TABLE I: In the above table, we tabulate the values of masses $m$, decay constants $f$ and threshold parameters $s_{0}$, of vector and axial-vector mesons.

62]. As we shall discuss later, the shift in masses and decay constants of vector and axialvector mesons are not much sensitive to the values of coupling constants and therefore, we used the same values for all vector and axial vector mesons under investigation [40, 51], i.e., we use, $g_{D^{*} N \Lambda_{c}} \approx g_{D^{*} N \Sigma_{c}} \approx g_{D_{1} N \Lambda_{c}} \approx g_{D_{1} N \Sigma_{c}} \approx g_{B^{*} N \Lambda_{b}} \approx g_{B^{*} N \Sigma_{b}} \approx g_{B_{1} N \Lambda_{b}} \approx g_{B_{1} N \Sigma_{b}}$ equal to 3.86 [40]. The masses of quarks namely, up $(u)$, down $(d)$, strange $(s)$, charm $(c)$, and bottom $(b)$, used in the present work are, 0.005, 0.007, 0.095, 1.35 and $4.7 \mathrm{GeV}$, respectively.

In Table I, we mentioned the values of masses $m$, decay constants $f$ and threshold parameters $s_{0}$, of vector and axial-vector mesons [40,51]. The values of decay constants of strange vector mesons are calculated in terms of decay constants of non-strange vector mesons in Refs. [50, 63 65]. In Ref. [50], using QCD sum rules, the values for $f_{D_{s}}{ }^{*}$ and $f_{B_{s}}{ }^{*}$ are found to be $1.21 f_{D^{*}}$ and $1.20 f_{B^{*}}$, respectively. In [63], the decay constants of $f_{D_{s}}$ are calculated from the simulations of twisted mass QCD and is observed to be $1.16 f_{D^{*}}$. The values of $f_{B_{s}{ }^{*}}$ in [65] is observed to be $1.16 f_{B^{*}}$. In our present calculations, for strange vector and axialvector mesons, we used the values 1.16 times the decay constant of respective non-strange meson, i.e., the values of $f_{D_{s}^{*}}, f_{B_{s}^{*}}, f_{D_{1 s}}$ and $f_{B_{1 s}}$ are $1.16 f_{D^{*}}, 1.16 f_{B^{*}}, 1.16 f_{D_{1}}$, and $1.16 f_{B_{1}}$, respectively [63, 65]. The continuum threshold parameter $s_{0}$, define the scale below which the continuum contribution vanishes [57]. The values of continuum threshold parameters $s_{0}$ are chosen so as to reproduce the experimental observed vacuum values of the masses of vector and axial-vector mesons [40, 51]. We assume that the various coupling constants and continuum threshold parameters are not subjected to medium modifications. 


\begin{tabular}{|c|c|c|c|c|c|c|}
\hline & $D^{*+}$ & $D^{* 0}$ & $D_{s}^{*}$ & $B^{*+}$ & $B^{* 0}$ & $B_{s}^{*}$ \\
\hline$\delta m$ & $(4.5-6.5)$ & $(4.5-6.5)$ & $(5.0-7.0)$ & $(30-33)$ & $(30-33)$ & $(31-34)$ \\
\hline$\delta f$ & $(3.3-4.9)$ & $(3.3-4.9)$ & $(3.8-5.3)$ & $(26-31)$ & $(26-31)$ & $(27-31)$ \\
\hline \hline & $D_{1}^{+}$ & $D_{1}^{0}$ & $D_{1 s}$ & $B_{1}^{+}$ & $B_{1}^{0}$ & $B_{1 s}$ \\
\hline$\delta m$ & $(5.4-9.4)$ & $(5.4-9.4)$ & $(5.9-9.9)$ & $(33-38)$ & $(33-38)$ & $(36-40)$ \\
\hline$\delta f$ & $(4.2-7.2)$ & $(4.2-7.2)$ & $(5.0-8.0)$ & $(30-34)$ & $(30-34)$ & $(32-36)$ \\
\hline
\end{tabular}

TABLE II: In the above table, we tabulate the Borel windows i.e. range of squared Borel mass parameter $M^{2}$, within which shift in mass $\delta m$ and decay constant $\delta f$ remain stable. The values shown in table are in $\mathrm{GeV}^{2}$ units and are given for both vector and axial-vector mesons.

To describe the exact mass (decay) shift of above mesons we have chosen a suitable Borel window, i.e., the range of squared Borel mass parameter $M^{2}$, within which there is almost no variation in the mass and decay constant. In Table II, we mentioned the Borel windows, as observed in the present calculations for the mass shift and shift in decay constant of vector and axial-vector mesons. In our calculations, we observe that, masses and decay constant of vector and axial-vector mesons are stable in different regions of $M^{2}$ and therefore, different Borel windows for masses and decay constant of respective mesons are mentioned in Table II. This is consistent with observations of Ref. [51]. Our further discussion in the present section is divided into three subsections. In subsection $A$, we shall discuss about the behavior of scalar fields and condensates in the strange hadronic medium, at zero and finite temperature. Subsections $B$ and $C$ will be devoted to present the results on vector and axial-vector mesons, respectively.

\section{A. Scalar Fields and Condensates:}

We start with the discussion on the behavior of quark and gluon condensates for different strangeness fractions and isospin asymmetry parameters of strange hadronic medium. In literature, the quark condensates are evaluated to leading order in nuclear density using the Feynman Hellmann theorem and model independent results were obtained in terms of pion nucleon sigma term [66, 67]. Using the Feynman Hellmann theorem, the quark condensate at finite density of nuclear matter is expressed as a sum of vacuum value and a term dependent 
on energy density of nuclear matter. In the model independent calculations, the interactions between nucleons were neglected and free space nucleon mass was used. If one use only the leading order calculations for the evaluation of quark condensates above nuclear matter density, then the quark condensates decreases very sharply and almost vanishes around $3 \rho_{0}$. In Ref. [68], the Dirac-Brueckner approach with the Bonn boson-exchange potential was used to include the higher order corrections and to find the quark condensates in the nuclear matter above the nuclear matter density. The calculations show that at higher density the quark condensates decrease more slowly as compared to leading order predictions.

As discussed earlier, in the chiral SU(3) model, used in the present work, the quark and gluon condensates are expressed in terms of scalar fields $\sigma, \zeta, \delta$ and $\chi$ [55, 58]. In Fig. 1, we show the variation of, ratio of in-medium value to vacuum value of scalar fields, as a function of baryonic density of strange hadronic medium. We show the results for temperatures $T=0$ and $T=100 \mathrm{MeV}$. For each value of temperature $T$, the results are plotted for isospin asymmetry parameters $I=0$ and 0.5 and the strangeness fractions $f_{s}=0$ and 0.5 . From Fig. 1, we can see that, the scalar fields $\sigma$ and $\zeta$ varies considerably as a function of baryonic density of medium, whereas the scalar field $\chi$ has little density dependence. For example, in symmetric nuclear medium $\left(I=0\right.$ and $\left.f_{s}=0\right)$, at density $\rho_{B}=\rho_{0}\left(4 \rho_{0}\right)$, the values of scalar fields $\sigma, \zeta$ and $\chi$, are observed to be $0.64 \sigma_{0}\left(0.31 \sigma_{0}\right), 0.91 \zeta_{0}\left(0.86 \zeta_{0}\right)$, and $0.99 \chi_{0}$ $\left(0.97 \chi_{0}\right)$. The symbols, $\sigma_{0}, \zeta_{0}$ and $\chi_{0}$ denote the vacuum values of scalar fields and have values $-93.29,-106.75$ and $409.76 \mathrm{MeV}$, respectively. It is observed that, at high baryon density, the strange scalar-isoscalar field $\zeta$, varies considerably as a function of strangeness fraction $f_{s}$, as compared to non-strange scalar isoscalar field $\sigma$. For example, in symmetric medium $(I=0)$, at baryon density $4 \rho_{0}$, as we move from $f_{s}=0$ to $f_{s}=0.5$, the value of $\zeta$ changes by $14 \%$, whereas the value of $\sigma$ changes by $1 \%$ only. However, the effect of isospin asymmetry of the medium is more on the values of $\sigma$ field as compared to $\zeta$ field. For example, in nuclear medium $\left(f_{s}=0\right)$, at baryon density $\rho_{B}=4 \rho_{0}$, as we move from $I$ $=0$ to 0.5 , the values of non-strange scalar field $\sigma$ and the strange scalar field $\zeta$ changes by $10.25 \%$ and $0.24 \%$ respectively. However, in strange medium, at $f_{s}=0.5$, the percentage change in the values of $\sigma$ and $\zeta$ is $7.2 \%$ and $7 \%$ respectively. Since the scalar meson $\sigma$, has light quark content ( $u$ and $d$ quarks) and the $\zeta$ meson have strange quark content ( $s$ quark) and therefore, former is more sensitive to isospin asymmetry of the medium (property of $u$ and $d$ quarks) and the latter is to the strangeness fraction. For the finite baryonic density 
of the medium, the scalar fields undergo less drop at non-zero temperature of the medium, as compared to zero temperature situation. This effect of temperature is more pronounced at finite strangeness and higher baryonic density of the medium. For example, in symmetric nuclear matter, at baryonic density $\rho_{B}=\rho_{0}$, for a change of temperature from $T=0$ to $T=100 \mathrm{MeV}$, the magnitude of scalar field $\sigma$, is increased by approximately $6 \%$. However, at $\rho_{B}=4 \rho_{0}$ and $f_{s}=0.5$, the increase in the magnitude of $\sigma$ is $12 \%$. The observed behavior of scalar fields with the temperature of the medium, in the present chiral SU(3) hadronic model, is consistent with the calculation within chiral quark mean field model discussed in Ref. [69].

In Figs. 2 and 3, the ratios of, in-medium value to the vacuum value of scalar quark and gluon condensates respectively, are plotted as a function of baryonic density of hadronic medium. We show the results for light quark condensates, $\langle\bar{u} u\rangle,\langle\bar{d} d\rangle$, the strange quark condensate, $\langle\bar{s} s\rangle$ and the gluon condensates, $\left\langle\frac{\alpha_{s}}{\pi} G_{\mu \nu}^{a} G^{\mu \nu a}\right\rangle$. In Table III, we tabulate the values of the ratio of these condensates for quantitative understanding. In this table, $\langle\bar{u} u\rangle_{0}$, $\langle\bar{d} d\rangle_{0}$ and $\langle\bar{s} s\rangle_{0}$, denotes the vacuum values of quark condensates and in chiral SU(3) model these are, $-1.401 \times 10^{-2} \mathrm{GeV}^{3},-1.401 \times 10^{-2} \mathrm{GeV}^{3}$ and $-4.671 \times 10^{-2} \mathrm{GeV}^{3}$ respectively. We observe that, for a given value of temperature $T$, isospin asymmetry parameter $I$, and the strangeness fraction $f_{s}$, as a function of baryonic density, the magnitude of the values of quark condensate decreases w.r.t. vacuum value. This is because, the values of quark condensates are proportional to the scalar fields $\sigma$ and $\zeta$ (see Eqs. (3) to (5) ). As discussed above, the magnitude of these scalar fields undergo drop as a function of density of baryonic matter and this further cause a decrease in the magnitude of quark condensates. As we move from symmetric to the asymmetric medium, due opposite contribution of scalar-isovector mesons $\delta$, the values of condensate $\langle\bar{u} u\rangle$ increases, whereas that of $\langle\bar{d} d\rangle$ decreases.

In isospin symmetric medium $(I=0)$, below baryon density $\rho_{B}=3.3 \rho_{0}$, as we move from non-strange medium, i.e., $f_{s}=0$, to strange medium with $f_{s}=0.5$, the magnitude of light quark condensates $\langle\bar{u} u\rangle$ increases. Above baryon density, $\rho_{B}=3.3 \rho_{0}$, the magnitude of the values of light quark condensates is more in non strange medium $\left(f_{s}=0\right)$ as compared to strange medium with finite $f_{s}$. From Fig. 2, we observe that, as a function of density of baryonic matter, we always observe a decrease in the values of quark condensates. This support the expectation of chiral symmetry restoration at high baryonic density. However, in linear Walecka model [70, 71] and also in Dirac-Brueckner approach [68], the values of 

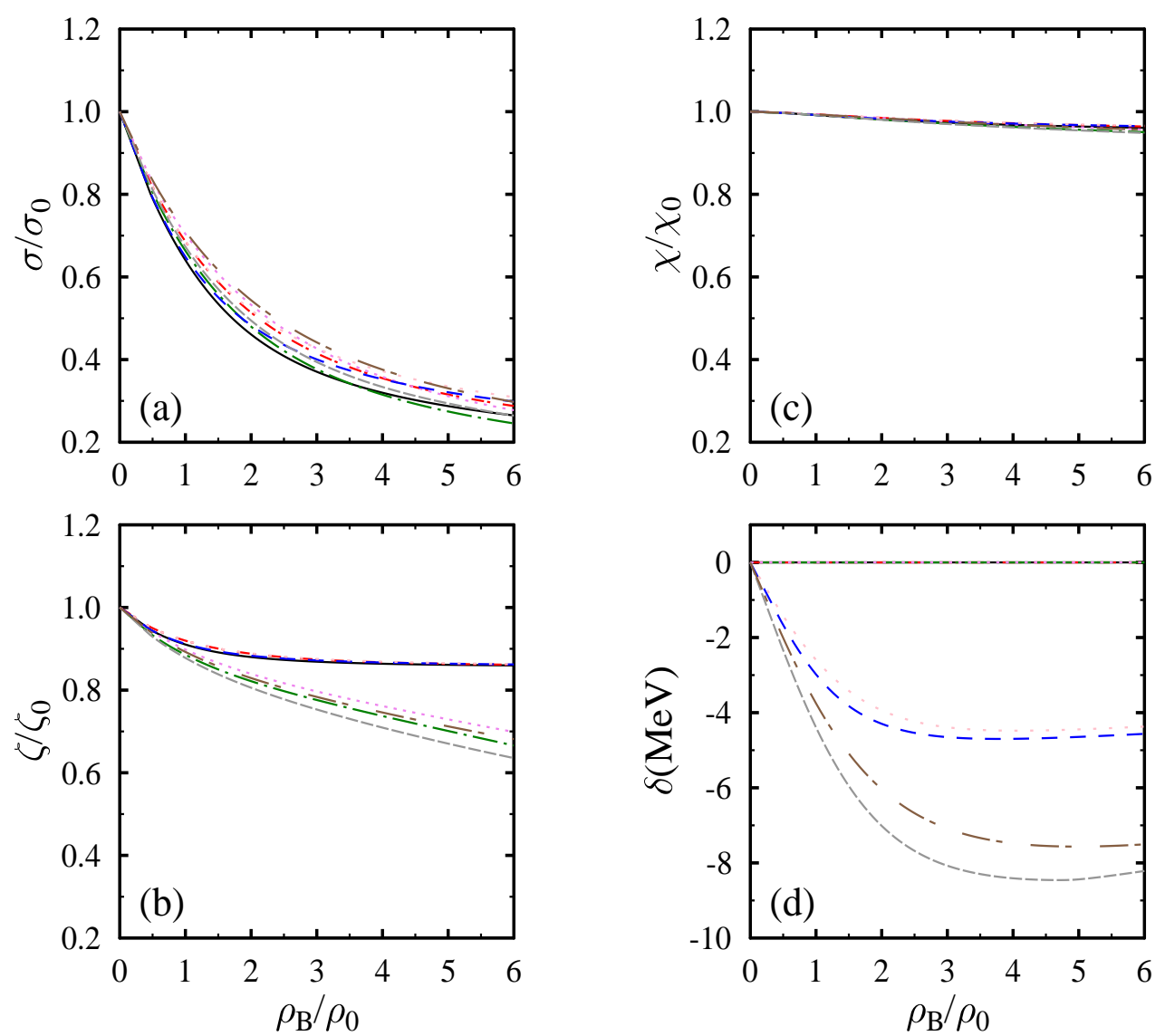

$$
\begin{array}{ll}
-\mathrm{I}=0 ; \mathrm{fs}=0 ; \mathrm{T}=0 & -\cdot-\mathrm{I}=0 ; \mathrm{fs}=0 ; \mathrm{T}=100 \mathrm{MeV} \\
-\mathrm{I}=0 ; \mathrm{fs}=0.5 ; \mathrm{T}=0 & \cdots \mathrm{I}=0 ; \mathrm{fs}=0.5 ; \mathrm{T}=100 \mathrm{MeV} \\
-\mathrm{I}=0.5 ; \mathrm{fs}=0 ; \mathrm{T}=0 & \mathrm{I}=0.5 ; \mathrm{fs}=0 ; \mathrm{T}=100 \mathrm{MeV} \\
--\mathrm{I}=0.5 ; \mathrm{fs}=0.5 ; \mathrm{T}=0-\mathrm{I}=0.5 ; \mathrm{fs}=0.5 ; \mathrm{T}=100 \mathrm{MeV}
\end{array}
$$

FIG. 1: (Color online) In the above figure, subplots (a), (b) and (c) show the variation of ratio of in-medium value to vacuum value of scalar fields, $\sigma / \sigma_{0}, \zeta / \zeta_{0}$ and $\chi / \chi_{0}$ as a function of baryonic density, $\rho_{B}$ (in units of nuclear saturation density, $\rho_{0}$ ). In subplot $(\mathrm{d})$ we have shown the scalarisovector field $\delta$ as a function of density of medium. We show the results at temperatures, $T$ $=0$ and $100 \mathrm{MeV}$. For each value of temperature, the results are plotted for isospin asymmetry parameters, $I=0$ and 0.5 and the strangeness fractions, $f_{s}=0$ and 0.5 .

quark condensates are observed to increase at higher values of baryonic density and causes hindrance to chiral symmetry restoration. The possible reason for this may be that the chiral invariance property was not considered in these calculations [68]. As the strange quark condensates $\langle\bar{s} s\rangle$, is proportional to the strange scalar-isoscalar field $\zeta$, therefore, the behavior of this field as a function of various parameters of the medium is also reflected 

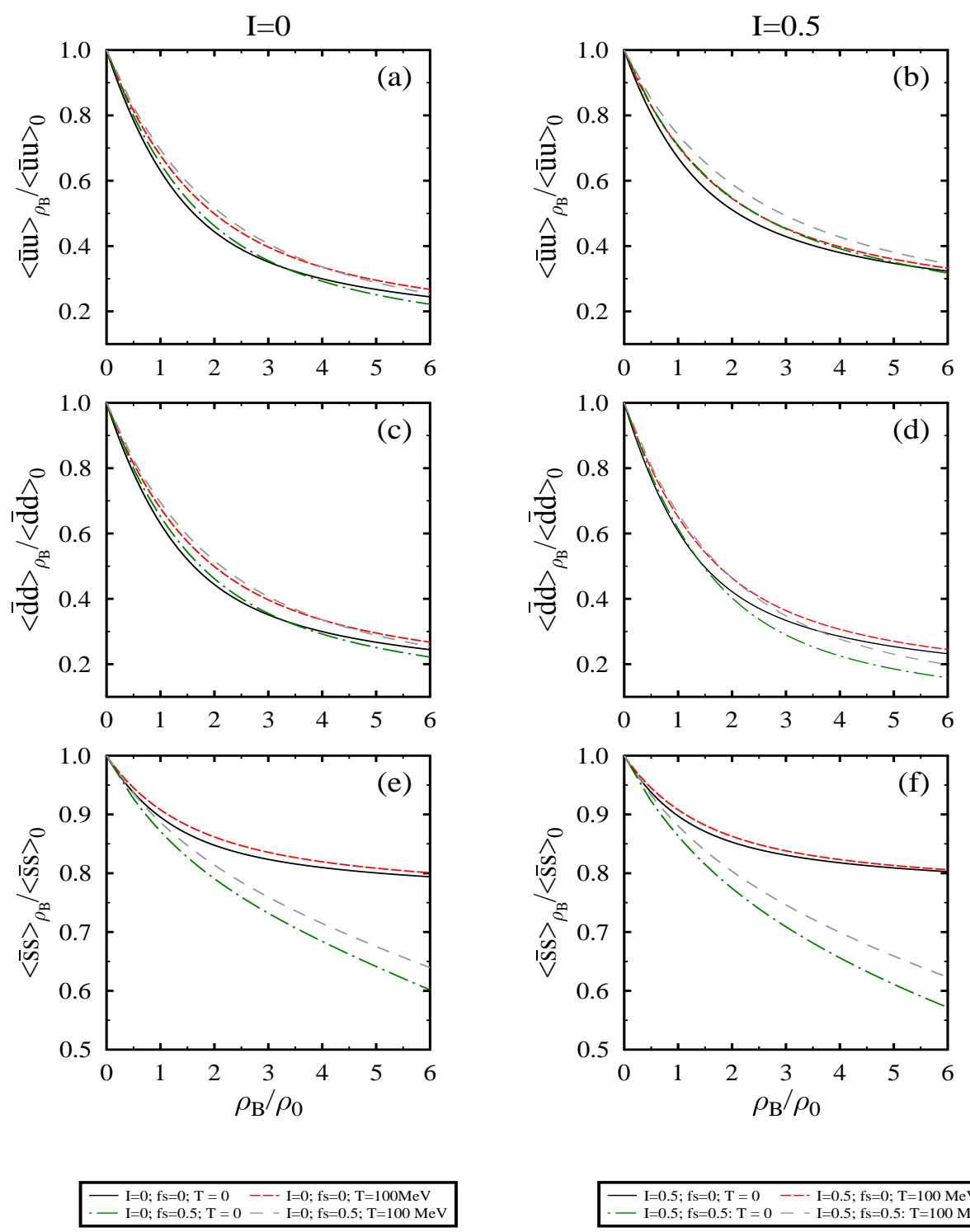

$-\mathrm{I}=0.5 ; \mathrm{fs}=0 ; \mathrm{T}=0 \quad---\mathrm{I}=0.5 ; \mathrm{fs}=0 ; \mathrm{T}=100 \mathrm{MeV}$
- I $=0.5 ; \mathrm{fs}=0.5: \mathrm{T}=0--\mathrm{I}=0.5 ; \mathrm{fs}=0.5: \mathrm{T}=100 \mathrm{MeV}$

FIG. 2: (Color online) In the above figure, we plot the ratio of in-medium quark condensate to vacuum condensate, as a function of baryonic density, $\rho_{B}$ (in units of nuclear saturation density, $\left.\rho_{0}\right)$. We compare the results at isospin asymmetric parameters $I=0$ and 0.5 . For each value of isospin asymmetry parameter $I$, the results are shown for strangeness fractions $f_{s}=0$ and 0.5 and temperatures $T=0$ and $100 \mathrm{MeV}$.

in the values of strange quark condensate $\langle\bar{s} s\rangle$. For fixed baryon density, $\rho_{B}$ and isospin asymmetry parameter $I$, as we move from non-strange to strange hadronic medium, the values of strange condensate decreases. 


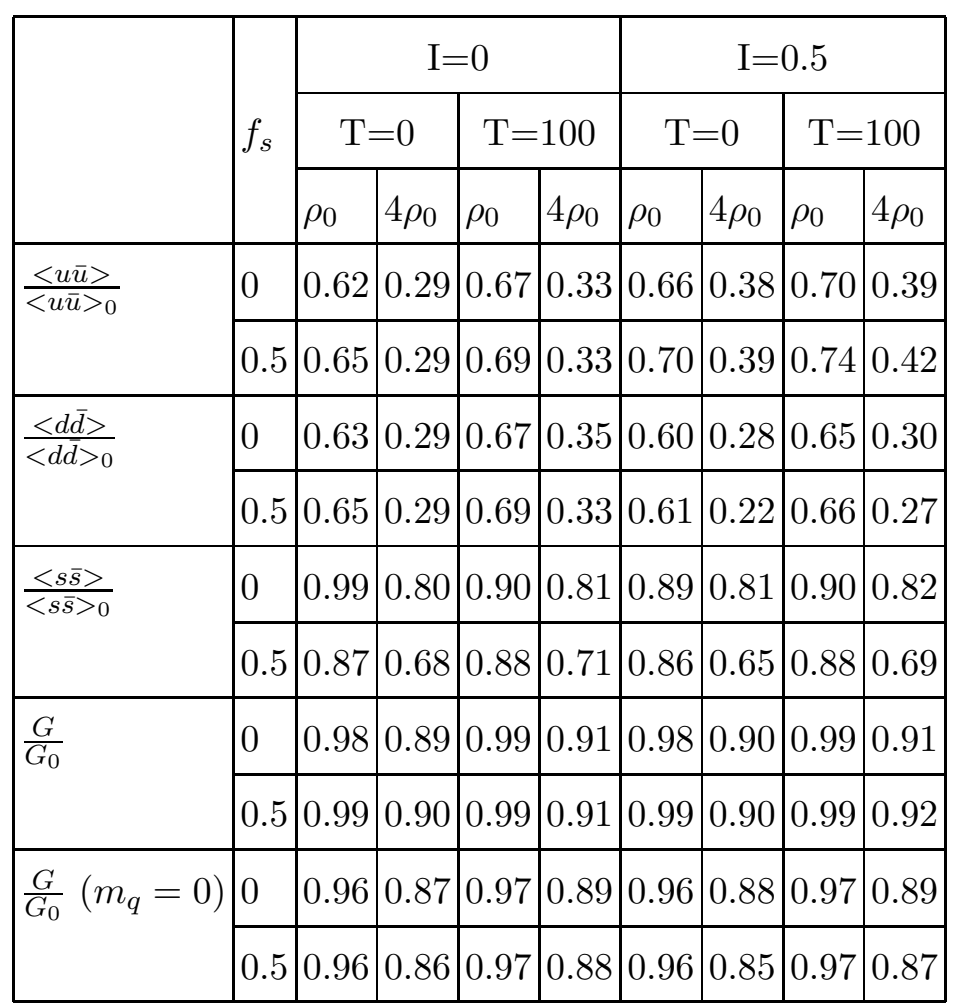

TABLE III: In the above table, we tabulate the ratio of in-medium value to the vacuum value of scalar quark and gluon condensates.

In Figs. 3(a) and 3(b), we consider the contribution of finite quark mass term in the calculation of gluon condensates, whereas Figs. 3)(c) and 3(d) are without the effect of quark mass term. We observe that, as a function of baryonic density of the hadronic medium, the values of gluon condensates decreases. As one can see from Figs. 3(c) and 3(d), the effect of strangeness fractions are more visible at higher baryon densities. The calculations show that the gluon condensates have small density dependence as compared to quark condensates. This observation is consistent with earlier model independent calculations by Cohen [67] and also with the QMC model calculation in Ref. [72].

Also it is observed that, at zero baryonic density, the behavior of scalar fields and hence the scalar quarks and gluon condensates, as a function of temperature of the medium is opposite to the situation of finite baryonic density. At $\rho_{B}=0$, the drop in the scalar fields and scalar condensates increases (magnitude decreases) with increase in temperature of the medium. Recall from previous discussion, at finite $\rho_{B}$, the drop in the scalar fields and hence scalar condensates was decreasing (magnitude increasing) with increase in the temperature of the medium (see Figs. 2 and 3). However, for zero baryonic density, the change in the 

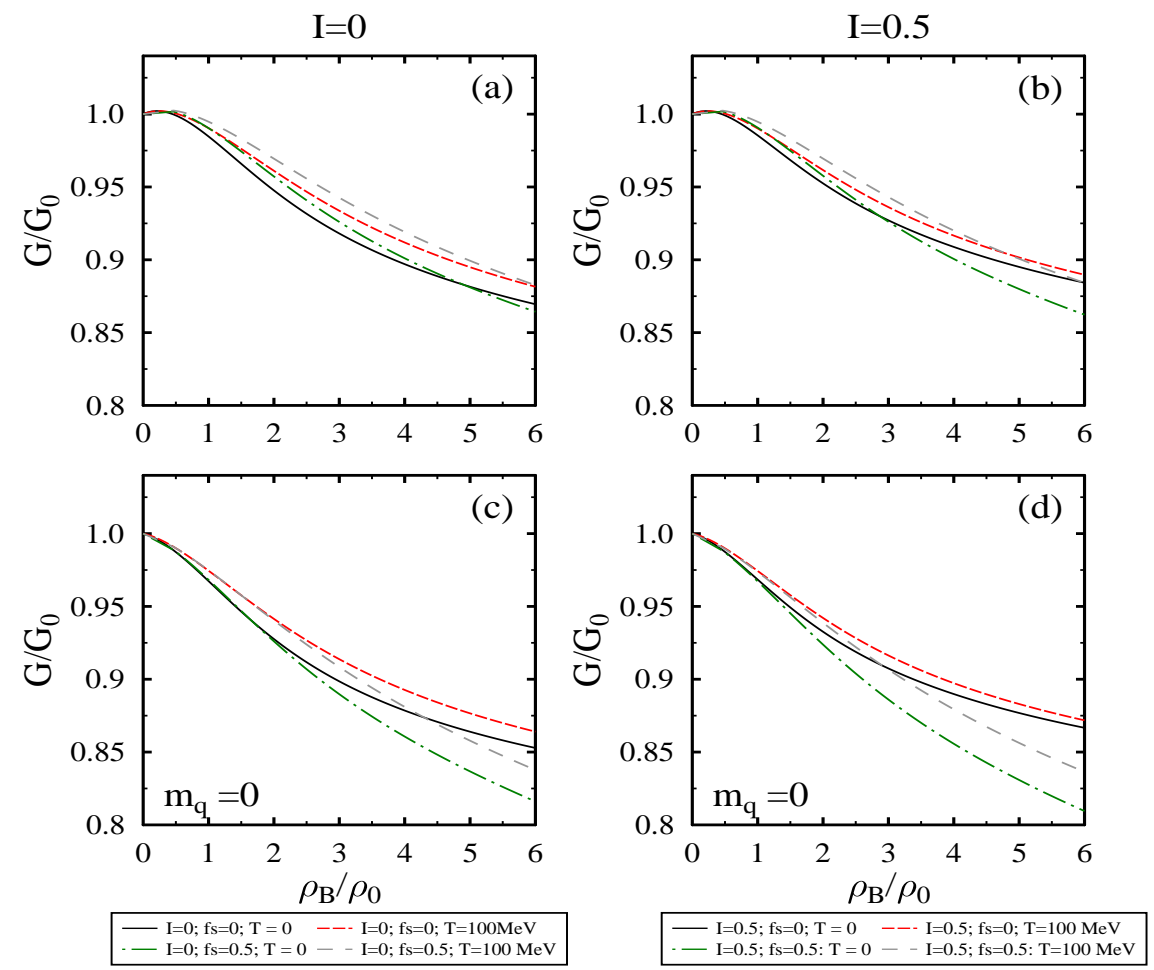

FIG. 3: (Color online) In the above figure, we plot the ratio of in-medium scalar gluon condensate to the vacuum value of gluon condensate, as a function of baryonic density, $\rho_{B}$ (in units of nuclear saturation density, $\left.\rho_{0}\right)$. We compare the results at isospin asymmetric parameters $I=0$ and 0.5 . For each value of isospin asymmetry parameter $I$, the results are shown for strangeness fractions $f_{s}$ $=0$ and 0.5 and temperatures $T=0$ and $100 \mathrm{MeV}$. In y-axis, $G=\left\langle\frac{\alpha_{s}}{\pi} G_{\mu \nu}^{a} G^{\mu \nu a}\right\rangle$ and $G_{0}$ denotes the expectation value of gluon condensate for zero density and temperature.

magnitude of scalar fields with the temperature is very small in the hadronic medium. For example, at $\rho_{B}=0$, the magnitude of the scalar field $\sigma(\zeta)$ decreases by $0.06 \%(0.02 \%)$ as one move from $T=0$ to $T=100 \mathrm{MeV}$. For a change of temperature from $T=100$ to $T=150 \mathrm{MeV}$, the magnitude of $\sigma(\zeta)$ decreases by $2.5 \%(0.8 \%)$. The values of, light quark condensate, strange quark condensate, and the gluon condensate with zero quark mass term, at $\rho_{B}=0$, changes by $2.75 \%, 0.85 \%$, and $0.08 \%$ respectively, as $T$ is changed from 0 to 150 $\mathrm{MeV}$. However, at very high temperature, possibly above critical temperature, the values of scalar condensates may change sharply [73]. As we discussed earlier, in literature the values of scalar condensates at finite temperatures and zero baryonic densities are calculated using the pion bath contributions [57]. Our observations on the behavior of condensates as a 


\begin{tabular}{|c|c|c|c|c|c|c|c|c|c|}
\hline \multirow{5}{*}{} & \multirow{3}{*}{$f_{s}$} & \multicolumn{5}{|c|}{$\mathrm{I}=0$} & \multicolumn{4}{c|}{$\mathrm{I}=0.5$} \\
\cline { 2 - 9 } & & $\rho_{0}$ & $4 \rho_{0}$ & $\rho_{0}$ & $4 \rho_{0}$ & $\rho_{0}$ & $4 \rho_{0}$ & $\rho_{0}$ & $4 \rho_{0}$ \\
\hline \multirow{2}{*}{$\delta m_{D^{*+}}$} & 0 & -64 & -104 & -54 & -97 & -68 & -104 & -60 & -97 \\
\cline { 2 - 9 } & 0.5 & -74 & -106 & -64 & -98 & -83 & -119 & -72 & -110 \\
\hline$\delta m_{D^{* 0}}$ & 0 & -92 & -160 & -79 & -150 & -81 & -132 & -71 & -132 \\
\cline { 2 - 10 } & 0.5 & -108 & -164 & -94 & -152 & -88 & -135 & -77 & -126 \\
\hline \multirow{2}{*}{$\delta m_{B^{*+}}$} & 0 & -443 & -862 & -383 & -813 & -392 & -752 & -348 & -728 \\
\cline { 2 - 9 } & 0.5 & -527 & -896 & -462 & -838 & -437 & -758 & -388 & -713 \\
\hline$\delta m_{B^{* 0}}$ & 0 & -312 & -596 & -271 & -563 & -333 & -610 & -294 & -590 \\
\cline { 2 - 9 } & 0.5 & -370 & -618 & -326 & -580 & -414 & -682 & -364 & -635 \\
\hline
\end{tabular}

TABLE IV: In the above table, we tabulate the values of mass shift (in units of MeV) of heavy vector mesons $D^{*}\left(D^{*+}\right.$ and $\left.D^{* 0}\right)$ and $B^{*}\left(B^{*+}\right.$ and $\left.B^{* 0}\right)$.

function of temperature, for zero baryonic density, are in accordance with the earlier findings [74 76$]$.

\section{B. Heavy Vector Mesons $\left(D^{*}, B^{*}, D_{s}^{*}\right.$ and $\left.B_{s}^{*}\right)$ :}

In Fig. 4 (Fig. 5), we show the variation of mass shift (shift in decay constant) of vector mesons $D^{*}$ and $B^{*}$, as a function of squared Borel mass parameter $M^{2}$. In each subplot, we compare the results for temperatures $T=0$ and $T=100 \mathrm{MeV}$. For each value of temperature, the results are plotted for isospin asymmetry parameters $I=0\left(f_{s}=0\right.$ and 0.5$)$ and $I=0.5\left(f_{s}=0\right.$ and 0.5$)$. We present the results at baryonic densities $\rho_{0}$ and $4 \rho_{0}$. In Table IV (Table $\mathrm{V}$ ), we have written the values of shift in mass (decay constant) of these vector mesons. The difference in the masses of $D^{*+}\left(B^{*+}\right)$ and $D^{* 0}\left(B^{* 0}\right)$ mesons in the symmetric nuclear medium is due to the different masses of $u$ and $d$ quarks, considered in the present investigation. Also we observe that, for a given value of baryonic density and strangeness fraction, both, the masses and decay constants, of $D^{*+}$ and $B^{* 0}\left(D^{* 0}\right.$ and $B^{*+}$ ) mesons, undergo more (less) drop in asymmetric medium as compared to symmetric medium. Note that, the $D^{*+}$ and $B^{* 0}$ mesons contain the light $d$ quark, whereas the $D^{* 0}$ 

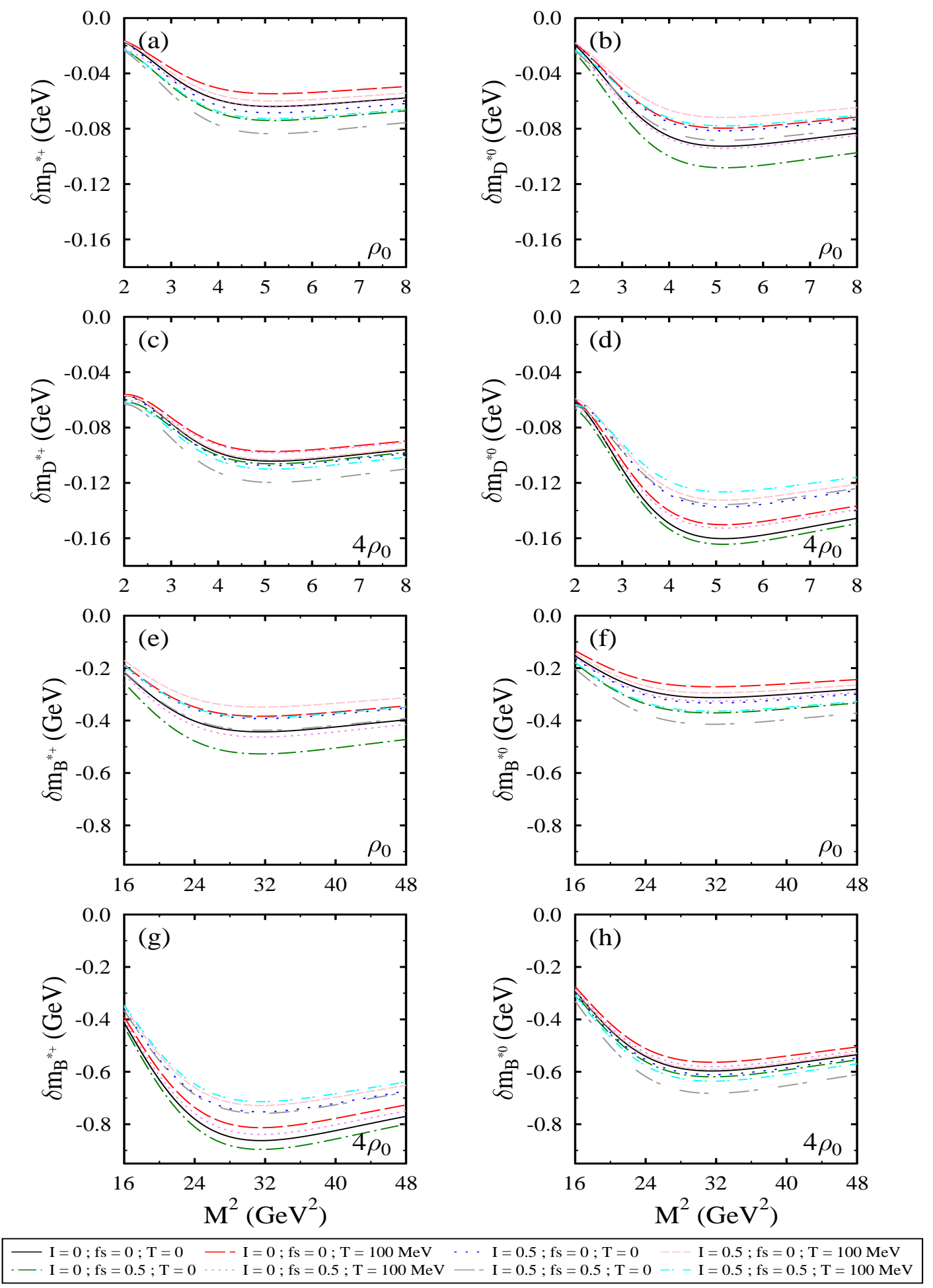

FIG. 4: (Color online) In the above figure, the variation of mass shift of vector mesons, $D^{*}\left(D^{*+}\right.$ and $\left.D^{* 0}\right)$ and $B^{*}\left(B^{*+}\right.$ and $\left.B^{* 0}\right)$, is shown as a function of squared Borel mass parameter $M^{2}$. We compare the results at temperatures $T=0$ and $T=100 \mathrm{MeV}$. For each value of temperature, the results are shown for isospin asymmetric parameters $I=0$ and 0.5 and the strangeness fractions $f_{s}=0$ and 0.5 . 


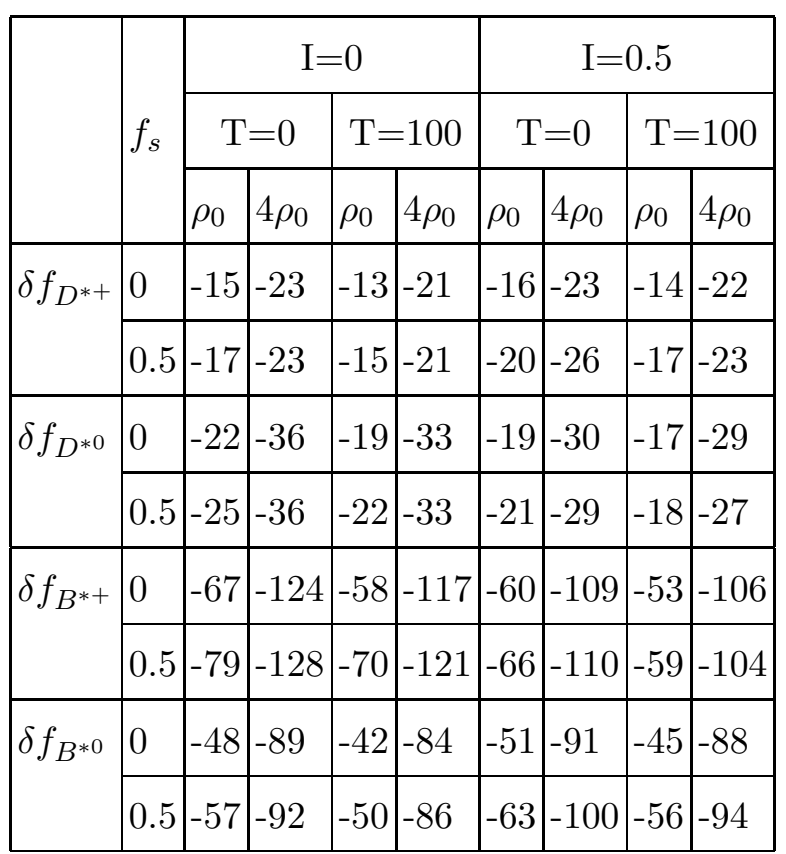

TABLE V: In the above table, we tabulate the values of shift in decay constant (in units of MeV) of heavy vector meson $D^{*}\left(D^{*+}\right.$ and $\left.D^{* 0}\right)$ and $B^{*}\left(B^{*+}\right.$ and $\left.B^{* 0}\right)$.

and $B^{*+}$ mesons have light $u$ quark. As discussed earlier, the behavior of $\langle\bar{d} d\rangle$ and $\langle\bar{u} u\rangle$ condensates is opposite as a function of asymmetry of the medium and this causes the observed behavior of $D^{*+}\left(B^{* 0}\right)$ and $D^{* 0}\left(B^{*+}\right)$ mesons, as a function of asymmetry of the medium. As compared to nuclear medium $\left(f_{s}=0\right)$, the drop in the masses and decay constant of $D^{*}$ and $B^{*}$ mesons is more in strange hadronic medium (finite $f_{s}$ ). Also, the drop in the masses of $D^{*}$ and $B^{*}$ mesons increases with increase in the baryonic density of the medium. At given finite baryonic density, the non-zero temperature of the medium cause less drop in the masses as well as decay constant of $D^{*}$ and $B^{*}$ mesons, as compared to zero temperature situation. This is because of increase in the values of scalar fields and condensates as a function of temperature of the medium, at given finite baryonic density [69].

In Fig. [6] and Table VI, we present the variations of mass shift and shift in decay constant of $D_{s}^{*}$ and $B_{s}^{*}$ mesons. The charmed strange and bottom strange mesons have one strange quark $s$ and one heavy quark. As discussed earlier, the in-medium properties of these mesons are calculated through the presence of strange quark condensate $\langle\bar{s} s\rangle$, in OPE side of QCD sum rule equations [40]. The negative values of shift in masses and decay constants show that these parameters of strange charmed and bottom vector meson undergo drop in the 

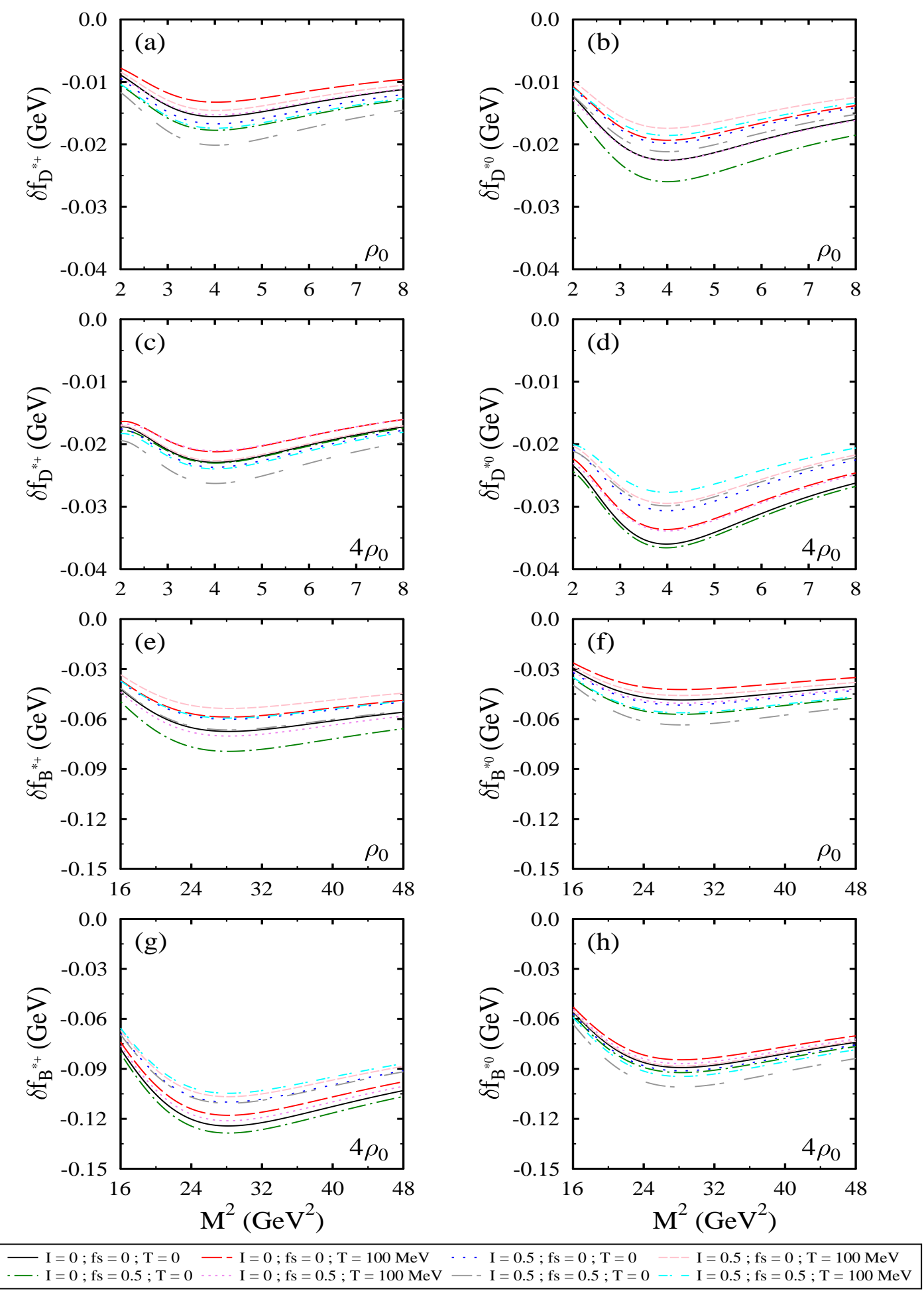

FIG. 5: (Color online) In the above figure, the variation of shift in decay constant of vector mesons $D^{*}\left(D^{*+}\right.$ and $\left.D^{* 0}\right)$ and $B^{*}\left(B^{*+}\right.$ and $\left.B^{* 0}\right)$, is shown as a function of squared Borel mass parameter $M^{2}$. We compare the results at temperatures $T=0$ and $T=100 \mathrm{MeV}$. For each value of temperature, the results are shown for isospin asymmetric parameters $I=0$ and 0.5 , and the strangeness fractions $f_{s}=0$ and 0.5 . 

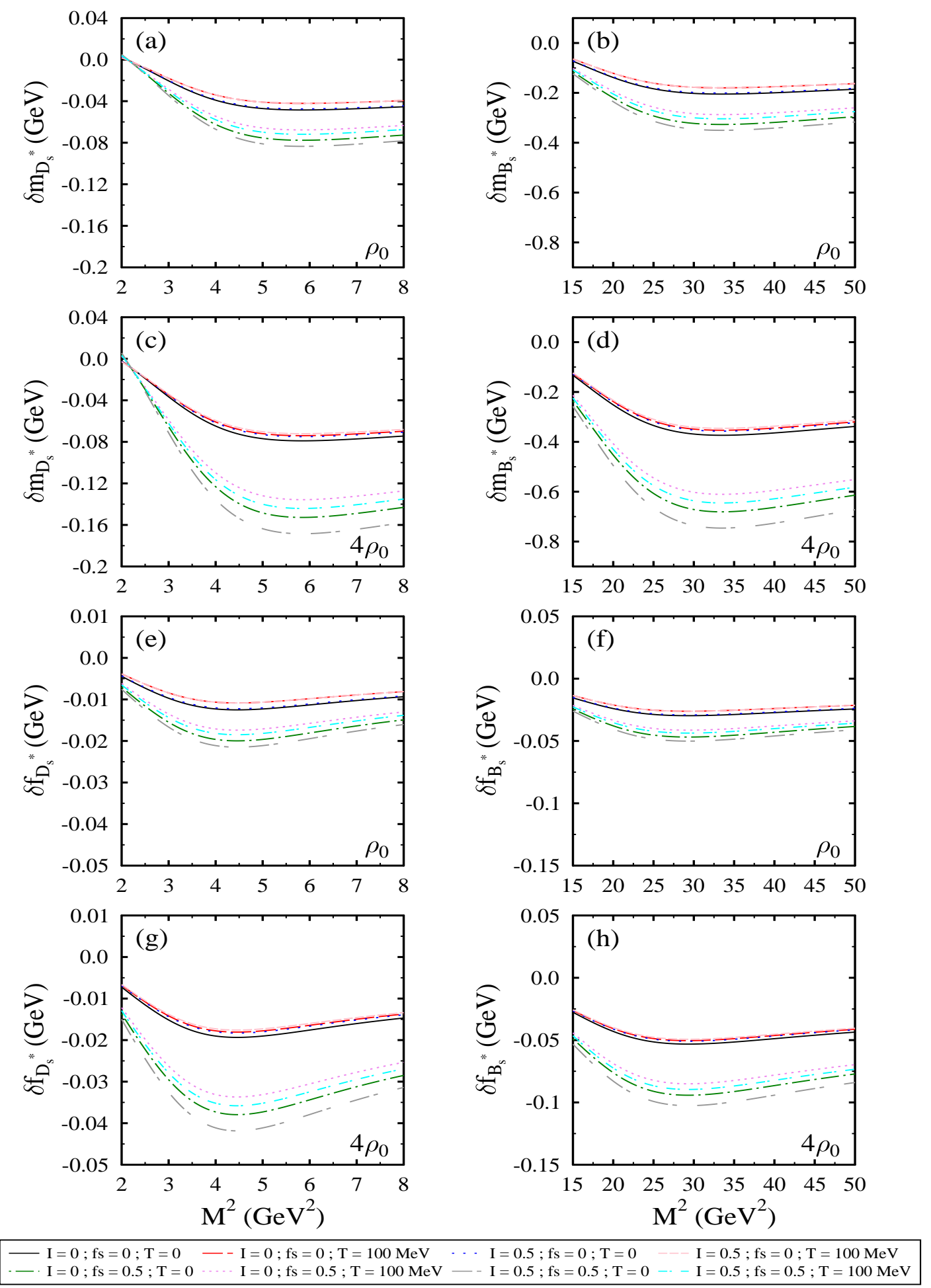

FIG. 6: (Color online) In the above figure, the variation of shift in mass and decay constant of strange vector mesons $D_{s}^{*}$ and $B_{s}^{*}$, is shown as a function of squared Borel mass parameter $M^{2}$. We show the results at temperatures $T=0$ and $T=100 \mathrm{MeV}$. For each value of temperature, the results are shown for isospin asymmetry parameter, $I=0$ and 0.5 and the strangeness fractions, $f_{s}=0$ and 0.5 . 


\begin{tabular}{|c|c|c|c|c|c|c|c|c|c|}
\hline \multirow{2}{*}{} & \multirow{3}{*}{$f_{s}$} & \multicolumn{5}{|c|}{$\mathrm{I}=0$} & \multicolumn{4}{c|}{$\mathrm{I}=0.5$} \\
\cline { 3 - 9 } & & \multicolumn{2}{|c|}{$\mathrm{T}=0$} & \multicolumn{2}{c|}{$\mathrm{T}=100$} & \multicolumn{2}{c|}{$\mathrm{T}=0$} & \multicolumn{2}{c|}{$\mathrm{T}=100$} \\
\cline { 3 - 10 } & $\rho_{0}$ & $4 \rho_{0}$ & $\rho_{0}$ & $4 \rho_{0}$ & $\rho_{0}$ & $4 \rho_{0}$ & $\rho_{0}$ & $4 \rho_{0}$ \\
\hline$\delta m_{D_{s}^{*}}$ & 0 & -48 & -79 & -42 & -74 & -47 & -74 & -42 & -72 \\
\cline { 2 - 10 } & 0.5 & -77 & -152 & -67 & -135 & -83 & -168 & -71 & -144 \\
\hline$\delta m_{B_{s}^{*}}$ & 0 & -204 & -373 & -179 & -353 & -200 & -357 & -174 & -345 \\
\cline { 2 - 10 } & 0.5 & -326 & -680 & -287 & -610 & -350 & -745 & -304 & -645 \\
\hline$\delta f_{D_{s}^{*}}$ & 0 & -12 & -19 & -10 & -18 & -12 & -18 & -10 & -17 \\
\cline { 2 - 9 } & 0.5 & -19 & -37 & -17 & -33 & -21 & -41 & -18 & -35 \\
\hline$\delta f_{B_{s}^{*}}$ & 0 & -29 & -53 & -26 & -50 & -29 & -51 & -26 & -49 \\
\cline { 2 - 9 } & 0.5 & -46 & -94 & -41 & -85 & -50 & -102 & -43 & -89 \\
\hline
\end{tabular}

TABLE VI: In the above table, we tabulate the values of shift in masses and decay constants (in units of $\mathrm{MeV}$ ) of heavy strange vector mesons $D_{s}^{*}$ and $B_{s}^{*}$.

hadronic medium. We notice that, the effect of increasing either the density or strangeness fraction of the medium is to decrease the masses and decay constants of $D_{s}^{*}$ and $B_{s}^{*}$ mesons. However, as observed for $D^{*}$ and $B^{*}$ mesons, the masses and decay constants of $D_{s}^{*}$ and $B_{s}^{*}$ also increase with increase in temperature of the medium.

It may be noted that, among the various condensates present in the QCD sum rule equations, the scalar quark condensates $\langle\bar{q} q\rangle$, have largest contribution for the medium modification of $D$ and $B$ meson properties. For example, if all the condensates are set to zero, except $<\bar{q} q>$, then shift in mass (decay constant) for $D^{*+}$ meson in symmetric nuclear medium $\left(f_{s}=0\right.$ and $\left.I=0\right)$ is observed to be $-69(-17.8) \mathrm{MeV}$ for $\rho_{B}=\rho_{0}$ and can be compared to the values $-64(-15) \mathrm{MeV}$ evaluated in the presence of all condensates. Also, the condensate $<q^{\dagger} i D_{0} q>$ is not evaluated within chiral SU(3) model. In literature, the condensate $\left\langle q^{\dagger} i D_{0} q\right\rangle$ is available in terms of linear density formula only and hence same is used in our calculations. Dependence of in-medium properties of $D$ mesons on this condensate is negligible, at least upto linear density approximation, as was also found in literature [19, 39, 40, 51]. However, at high density it might become important. Neglecting $\left\langle q^{\dagger} i D_{0} q\right\rangle$ only, the values of mass shift (decay shift) in symmetric medium, at $\rho_{B}=\rho_{0}, 2 \rho_{0}$ and $4 \rho_{0}$ are observed to be $-62(-15.5),-89(-21)$ and $-97(-21) \mathrm{MeV}$, respectively. These values can 
be compared with the values, -64(-15), -92(-22) and -104(-23) MeV, respectively, which are computed in the situation when we considered the contribution of $\left\langle q^{\dagger} i D_{0} q>\right.$ within linear density approximation. Thus, the mass shift (decay shift) for $D^{\star+}$ mesons at density $2 \rho_{0}$ is changed by $3.58 \%(2.3 \%)$, whereas, at $4 \rho_{0}$, the percentage change is $6.7 \%(4.8 \%)$.

The observed modifications of masses and decay constant of vector mesons are not much sensitive to the values of coupling constant of heavy baryons with nucleons and charmed/bottom mesons. For example, in symmetric nuclear matter, at nuclear saturation density $\rho_{0}$, for an increase (decrease) of $20 \%$ in the value of coupling constant, the shift in the mass of $D^{*+}$ mesons decreases (increases) by $1.4 \%$ (2.8\%) only. As we discussed earlier, the continuum threshold parameters are chosen so as to reproduce the experimental observed vacuum values of the masses of charmed and bottom mesons mesons. The values of threshold parameter effect significantly the masses and decay constant of $D$ and $B$ mesons. For example, for a decrease (increase) of $10 \%$ in the values of threshold parameter $s_{0}$, in symmetric nuclear matter, at $\rho_{B}=\rho_{0}$, an increase (decrease) of $29 \%$ (15\%) is observed in the value of mass shift of $D^{*+}$ mesons.

\section{Heavy Axial-Vector Mesons $\left(D_{1}, B_{1}, D_{1 s}\right.$ and $\left.B_{1 s}\right)$ :}

Figures 7 and 8 show the variation of mass shift and shift in decay constant, respectively, of axial-vector mesons $\left[D_{1}\left(D_{1}^{0}\right.\right.$ and $\left.D_{1}^{+}\right)$and $B_{1}\left(B_{1}^{0}\right.$ and $\left.\left.B_{1}^{+}\right)\right]$, as a function of squared Borel mass parameter, i.e., $M^{2}$. In Tables VII and VIII, we tabulate the values of shift in mass and decay constants of these axial-vector mesons. Similarly, Fig. 9 and Table IX, are for the strange axial-vector, $D_{1 s}$ and $B_{1 s}$ mesons. For a constant value of strangeness fraction $f_{s}$, and isospin asymmetric parameter $I$, as a function of baryonic density, a positive shift in masses and decay constants of axial-vector mesons was observed. For a given density and isospin asymmetry, the finite strangeness fraction of the medium also causes an increase in the masses and decay constants of above axial-vector mesons. For the axial-vector $D_{1}$ and $B_{1}$ meson doublet, as a function of isospin asymmetry of the medium, the values of mass shift and decay shift of $D_{1}^{0}\left(B_{1}^{0}\right)$ meson decreases (increases), whereas that of $D_{1}^{+}$ $\left(B_{1}^{+}\right)$increases (decreases). As discussed earlier, in the case of vector mesons, the reason for opposite behavior of $D$ and $B$ mesons as a function of isospin asymmetry of medium is the presence of light $u$ quark in $D_{1}^{0}$ and $B_{1}^{+}$mesons, whereas the mesons $D_{1}^{+}$and $B_{1}^{0}$ have 


\begin{tabular}{|c|l|l|l|l|l|l|l|l|l|}
\hline \multirow{2}{*}{} & \multirow{3}{*}{$f_{s}$} & \multicolumn{4}{|c|}{$\mathrm{T}=0$} & \multicolumn{3}{c|}{$\mathrm{T}=100$} & \multicolumn{3}{c|}{$\mathrm{T}=0$} & \multicolumn{2}{c|}{$\mathrm{T}=100$} \\
\cline { 3 - 9 } & & $\rho_{0}$ & $4 \rho_{0}$ & $\rho_{0}$ & $4 \rho_{0}$ & $\rho_{0}$ & $4 \rho_{0}$ & $\rho_{0}$ & $4 \rho_{0}$ \\
\hline \multirow{2}{*}{$\delta m_{D_{1}^{+}}$} & 0 & 62 & 110 & 54 & 104 & 66 & 112 & 59 & 109 \\
\cline { 2 - 10 } & 0.5 & 72 & 113 & 64 & 106 & 81 & 124 & 72 & 116 \\
\hline \multirow{2}{*}{$\delta m_{D_{1}^{0}}$} & 0 & 88 & 158 & 168 & 149 & 78 & 140 & 69 & 134 \\
\cline { 2 - 9 } & 0.5 & 103 & 163 & 91 & 153 & 86 & 140 & 76 & 131 \\
\hline \multirow{2}{*}{$\delta m_{B_{1}^{+}}$} & 0 & 300 & 554 & 262 & 526 & 268 & 492 & 240 & 478 \\
\cline { 2 - 9 } & 0.5 & 353 & 573 & 313 & 541 & 297 & 497 & 266 & 470 \\
\hline \multirow{2}{*}{$\delta m_{B_{1}^{0}}$} & 0 & 216 & 401 & 189 & 380 & 229 & 409 & 204 & 396 \\
\cline { 2 - 9 } & 0.5 & 255 & 415 & 225 & 391 & 282 & 452 & 250 & 424 \\
\hline
\end{tabular}

TABLE VII: In the above table, we tabulate the values of mass shift (in units of MeV) of heavy axial-vector mesons $D_{1}\left(D_{1}^{+}\right.$and $\left.D_{1}^{0}\right)$ and $B_{1}\left(B_{1}^{+}\right.$and $\left.B_{1}^{0}\right)$.

light $d$ quark. At finite baryonic density, for nuclear as well as strange hadronic medium, the effect of finite temperature is to cause an increase in the values of positive mass shift of the axial-vector mesons w.r.t. zero temperature case.

The mass shift and shift in decay constants of charmed and bottom vector and axialvector mesons have been investigated in past using QCD sum rules in symmetric nuclear matter only [40, 51] . The values of mass shift for $D^{*}$ and $B^{*}$ mesons, in leading order (next to leading order) calculations, were $-70(-102)$ and $-340(-687) \mathrm{MeV}$, respectively. For the axial-vector $D_{1}$ and $B_{1}$ mesons, the above values of mass shift changes to 66 (97) and 260 (522) $\mathrm{MeV}$, respectively. The values of shift in decay constant for $D^{*}$ and $B^{*}$ mesons, in leading order (next to leading order), are found to be -18(-26) and -55(-111) MeV, whereas for $D_{1}$ and $B_{1}$ mesons these values changes to $21(31)$ and 67 (134) MeV, respectively. We can compare the above values of mass shift (decay shift) to our results -63 (-20), -312 (48.9), 62 (20) and $216(53) \mathrm{MeV}$ for $D^{*}, B^{*}, D_{1}$ and $B_{1}$ mesons, evaluated using $m_{u}=m_{d}$ $=7 \mathrm{MeV}$, in symmetric nuclear matter $\left(I=0\right.$ and $\left.f_{s}=0\right)$. As discussed earlier, in our calculations, for strange mesons, we used the value of decay constant equal to 1.16 times the decay constant of non-strange mesons. However, as an example, if we use, $f_{D_{s}}=1.19 f_{D}$, then the values of mass shift, at $\rho_{0}\left(I=0, f_{s}=0\right)$, for vector and axial-vector mesons, are 

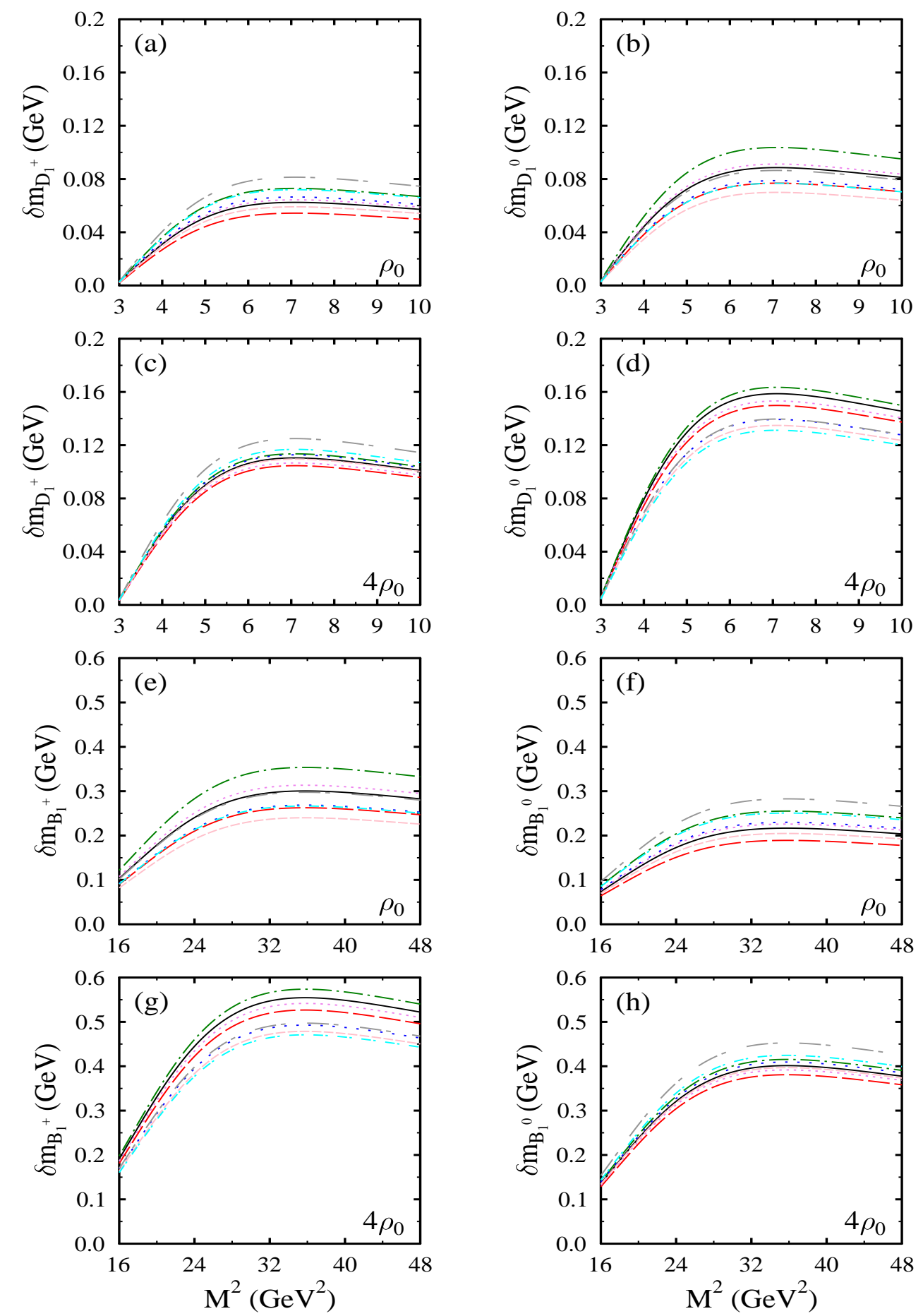

\begin{tabular}{|l}
$-\mathrm{I}=0 ; \mathrm{fs}=0 ; \mathrm{T}=0-\mathrm{I}=0 ; \mathrm{fs}=0 ; \mathrm{T}=100 \mathrm{MeV}-\mathrm{I}=0.5 ; \mathrm{fs}=0 ; \mathrm{T}=0 \quad-\mathrm{I}=0.5 ; \mathrm{fs}=0 ; \mathrm{T}=100 \mathrm{MeV}$ \\
$\mathrm{I}=0 ; \mathrm{fs}=0.5 ; \mathrm{T}=0-\mathrm{I}=0 ; \mathrm{fs}=0.5 ; \mathrm{T}=100 \mathrm{MeV}-\mathrm{I}=0.5 ; \mathrm{fs}=0.5 ; \mathrm{T}=0-\mathrm{I}=0.5 ; \mathrm{fs}=0.5 ; \mathrm{T}=100 \mathrm{MeV}$
\end{tabular}

FIG. 7: (Color online) In the above figure, the variation of mass shift of axial-vector mesons, $D_{1}$ $\left(D_{1}^{+}\right.$and $\left.D_{1}^{0}\right)$ and $B_{1}\left(B_{1}^{+}\right.$and $\left.B_{1}^{0}\right)$, is shown as a function of squared Borel mass parameter $M^{2}$. We compare the results at temperatures $T=0$ and $T=100 \mathrm{MeV}$. For each value of temperature, the results are shown for isospin asymmetric parameters $I=0$ and 0.5 and the strangeness fractions $f_{s}=0$ and 0.5 . 

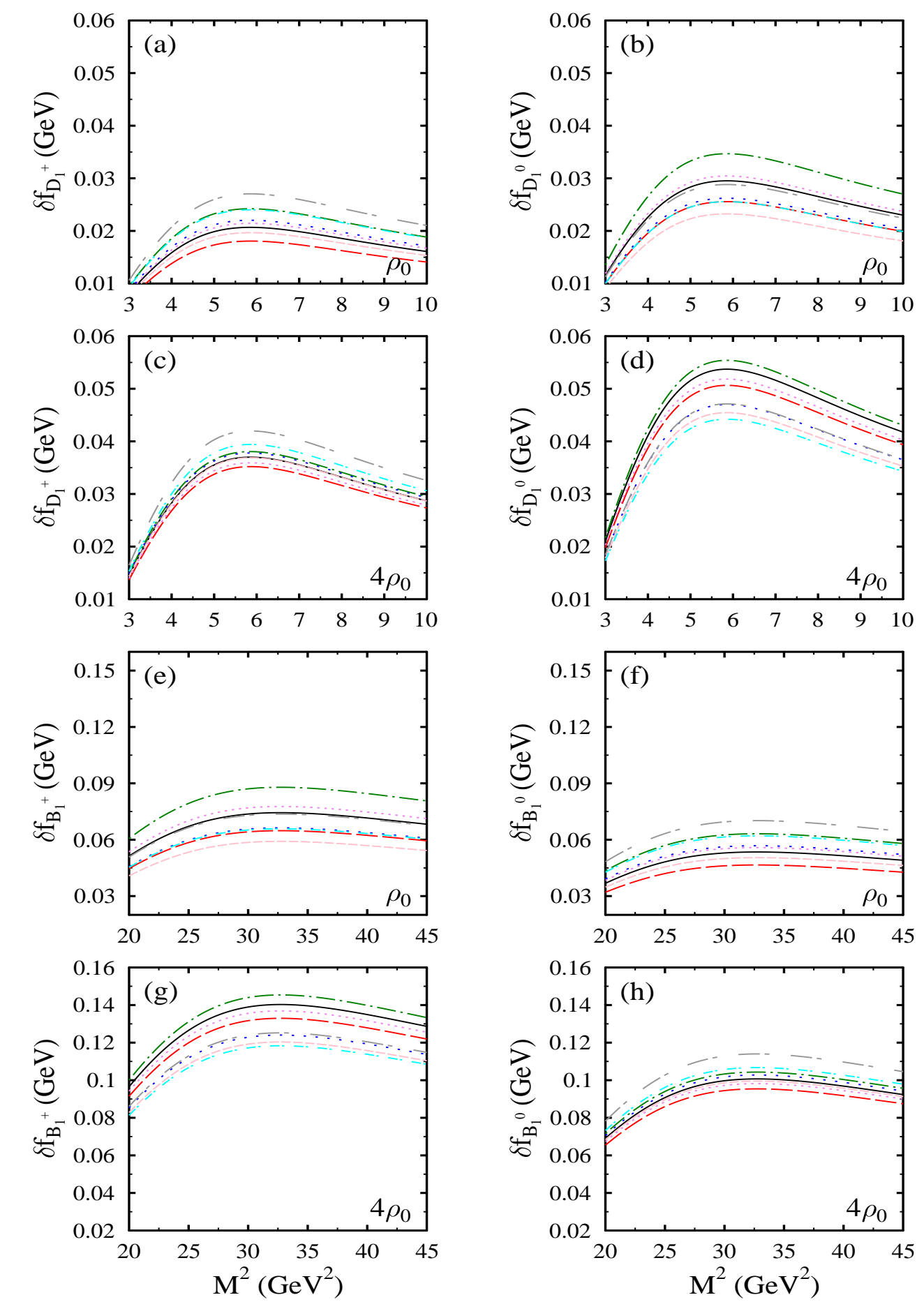

\begin{tabular}{|l}
$-\mathrm{I}=0 ; \mathrm{fs}=0 ; \mathrm{T}=0--\mathrm{I}=0 ; \mathrm{fs}=0 ; \mathrm{T}=100 \mathrm{MeV} \cdots \mathrm{I}=0.5 ; \mathrm{fs}=0 ; \mathrm{T}=0 \quad-\mathrm{I}=0.5 ; \mathrm{fs}=0 ; \mathrm{T}=100 \mathrm{MeV}$ \\
$-\mathrm{I}=0 ; \mathrm{fs}=0.5 ; \mathrm{T}=0-\mathrm{I}=0 ; \mathrm{fs}=0.5 ; \mathrm{T}=100 \mathrm{MeV}-\mathrm{I}=0.5 ; \mathrm{fs}=0.5 ; \mathrm{T}=0-\mathrm{I}=0.5 ; \mathrm{fs}=0.5 ; \mathrm{T}=100 \mathrm{MeV}$
\end{tabular}

FIG. 8: (Color online) In the above figure, the variation of shift in decay constant of axial-vector mesons, $D_{1}\left(D_{1}^{+}\right.$and $\left.D_{1}^{0}\right)$ and $B_{1}\left(B_{1}^{+}\right.$and $\left.B_{1}^{0}\right)$, is shown as a function of squared Borel mass parameter $M^{2}$. We compare the results at temperatures $T=0$ and $T=100 \mathrm{MeV}$. For each value of temperature, the results are shown for isospin asymmetric parameters $I=0$ and 0.5 and the strangeness fractions $f_{s}=0$ and 0.5 . 

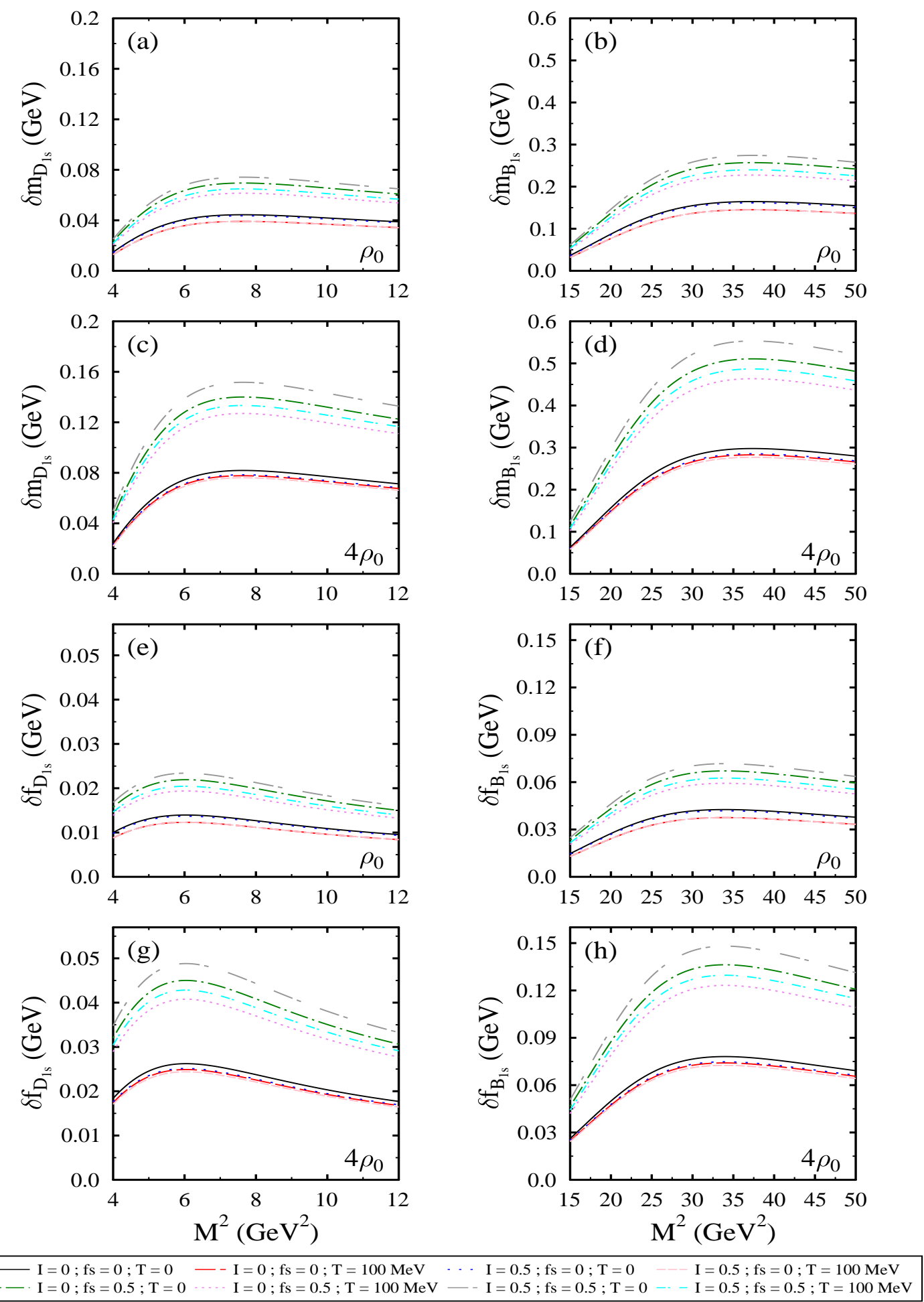

FIG. 9: (Color online) In the above figure, the variation of shift in mass and decay constant of strange axial-vector mesons $D_{1 s}$ and $B_{1 s}$, is shown as a function of squared Borel mass parameter $M^{2}$. We show the results at temperatures $T=0$ and $T=100 \mathrm{MeV}$. For each value of temperature, the results are shown for isospin asymmetry parameter, $I=0$ and 0.5 and the strangeness fractions, $f_{s}=0$ and 0.5 . 


\begin{tabular}{|l|l|l|l|l|l|l|l|l|l|}
\hline \multirow{2}{*}{} & \multirow{3}{*}{$f_{s}$} & \multicolumn{4}{|c|}{$\mathrm{T}=0$} & \multicolumn{3}{c|}{$\mathrm{T}=100$} & \multicolumn{3}{c|}{$\mathrm{T}=0$} & \multicolumn{2}{c|}{$\mathrm{T}=100$} \\
\cline { 2 - 10 } & & $\rho_{0}$ & $4 \rho_{0}$ & $\rho_{0}$ & $4 \rho_{0}$ & $\rho_{0}$ & $4 \rho_{0}$ & $\rho_{0}$ & $4 \rho_{0}$ \\
\hline \multirow{2}{*}{$\delta f_{D_{1}^{+}}$} & 0 & 20 & 36 & 18 & 35 & 22 & 37 & 19 & 36 \\
\cline { 2 - 10 } & 0.5 & 24 & 38 & 21 & 36 & 27 & 41 & 24 & 39 \\
\hline \multirow{2}{*}{$\delta f_{D_{1}^{0}}$} & 0 & 29 & 53 & 25 & 50 & 26 & 46 & 23 & 45 \\
\cline { 2 - 10 } & 0.5 & 34 & 55 & 30 & 51 & 28 & 47 & 25 & 44 \\
\hline \multirow{2}{*}{$\delta f_{B_{1}^{+}}$} & 0 & 74 & 140 & 64 & 132 & 66 & 124 & 59 & 120 \\
\cline { 2 - 10 } & 0.5 & 87 & 145 & 77 & 136 & 73 & 125 & 65 & 118 \\
\hline$\delta f_{B_{1}^{0}}$ & 0 & 53 & 100 & 46 & 95 & 56 & 102 & 50 & 99 \\
\cline { 2 - 10 } & 0.5 & 63 & 104 & 55 & 98 & 70 & 113 & 62 & 106 \\
\hline
\end{tabular}

TABLE VIII: In the above table, we tabulate the values of shift in decay constant (in units of $\mathrm{MeV}$ ) of heavy axial-vector mesons $D_{1}\left(D_{1}^{+}\right.$and $\left.D_{1}^{0}\right)$ and $B_{1}\left(B_{1}^{+}\right.$and $\left.B_{1}^{0}\right)$.

observed to be -46 and $44 \mathrm{MeV}$, respectively and can be compared with the values -48 and $42 \mathrm{MeV}$, respectively, obtained using, $f_{D_{s}}=1.16 f_{D}$.

In [20, 21], it was observed that beyond linear density approximation there occur strong splitting between the particle-antiparticle mass. In these work, the correlation function was parametrized, using the Lehmann representation, into the form, $\operatorname{Im} \pi(\omega, 0)=\Delta \pi(\omega)=$ $\pi F_{+} \delta\left(\omega-m_{+}\right)-\pi F_{-} \delta\left(\omega+m_{-}\right)$, such that, $m_{ \pm}=m \pm \Delta m$ and $F_{ \pm}=F \pm \Delta F$. Here, $m$ is mass center and $\Delta m$ is the mass splitting between particle-antiparticles. This correlation function was further used in the odd and even part of QCD sum rules. However, in our present approach, following the work of [19, 40], the correlation function is divided into the vacuum part and a medium dependent part and the average mass-shift was obtained. In Ref. [77], the mass-splitting between vector and axial-vector mesons had been studied using the Weinberg sum rules.

In the self consistent coupled channel approach of Ref. [16], the repulsive optical potential for $D^{*}$ mesons had been reported which is complementary to our calculations within QCD sum rules. The observed negative values of mass shift for $D^{*}$ and $B^{*}$ mesons in nuclear and strange hadronic matter, in our calculations, favor the decay of higher charmonium and bottomonium states to $D^{*} \bar{D}^{*}$ and $B^{*} \bar{B}^{*}$ pairs and hence may cause the quarkonium 


\begin{tabular}{|l|l|l|l|l|l|l|l|l|l|}
\hline \multirow{2}{*}{} & \multirow{3}{*}{$f_{s}$} & \multicolumn{4}{|c|}{$\mathrm{I}=0$} & \multicolumn{4}{c|}{$\mathrm{I}=0.5$} \\
\cline { 2 - 9 } & & \multicolumn{2}{|c|}{$\mathrm{T}=0$} & \multicolumn{2}{c|}{$\mathrm{T}=100$} & \multicolumn{2}{c|}{$\mathrm{T}=0$} & \multicolumn{2}{c|}{$\mathrm{T}=100$} \\
\cline { 2 - 10 } & $\rho_{0}$ & $4 \rho_{0}$ & $\rho_{0}$ & $4 \rho_{0}$ & $\rho_{0}$ & $4 \rho_{0}$ & $\rho_{0}$ & $4 \rho_{0}$ \\
\hline \multirow{2}{*}{$\delta m_{D_{1 s}}$} & 0 & 44 & 81 & 39 & 77 & 43 & 78 & 39 & 76 \\
\cline { 2 - 10 } & 0.5 & 69 & 140 & 61 & 127 & 74 & 151 & 64 & 133 \\
\hline \multirow{2}{*}{$\delta m_{B_{1 s}}$} & 0 & 164 & 297 & 145 & 282 & 161 & 285 & 145 & 276 \\
\cline { 2 - 10 } & 0.5 & 257 & 510 & 227 & 463 & 274 & 553 & 239 & 486 \\
\hline \multirow{2}{*}{$\delta f_{D_{1 s}}$} & 0 & 14 & 26 & 12 & 24 & 13 & 25 & 12 & 24 \\
\cline { 2 - 9 } & 0.5 & 21 & 45 & 19 & 40 & 23 & 48 & 20 & 42 \\
\hline$\delta f_{B_{1 s}}$ & 0 & 42 & 78 & 37 & 74 & 41 & 74 & 37 & 72 \\
\cline { 2 - 9 } & 0.5 & 67 & 136 & 59 & 123 & 71 & 148 & 62 & 129 \\
\hline
\end{tabular}

TABLE IX: In the above table, we tabulate the values of shift in masses and decay constants (in units of $\mathrm{MeV}$ ) of heavy strange axial-vector mesons $D_{1 s}$ and $B_{1 s}$.

suppression. However, the axial-vector meson undergo a positive mass shift in nuclear and strange hadronic medium and hence the possibility of decay of excited charmonium and bottomonium states to $D_{1} \bar{D}_{1}$ and $B_{1} \bar{B}_{1}$ pairs is suppressed. The observed effects of isospin asymmetry of the medium on the mass modifications of $D$ and $B$ mesons can be verified experimentally through the ratios $\frac{D^{*+}}{D^{* 0}}, \frac{B^{*+}}{B^{* 0}}, \frac{D_{1}^{+}}{D_{1}^{0}}$ and $\frac{B_{1}^{+}}{B_{1}^{0}}$, whereas the effects of strangeness of the matter can be seen through the ratios $\frac{D^{*}}{D_{S}^{*}}, \frac{B^{*}}{B_{S}^{*}}, \frac{D_{1}}{D_{1 S}}$ and $\frac{B_{1}}{B_{1 S}}$. The traces of observed medium modifications of masses and decay constants can be seen experimentally in the strong decay width and leptonic decay width of heavy mesons [52]. For example, in Ref. [43] the couplings $g_{D^{*} D \pi}$ and $g_{B^{*} B \pi}$ were studied using the QCD sum rules and strong decay width of charged vector $D^{*+}$ mesons for the strong decay, $D^{*+} \longrightarrow D^{0} \pi^{+}$were evaluated using the formula,

$$
\Gamma\left(D^{*} \longrightarrow D \pi\right)=\frac{g_{D^{*} D \pi}^{2}}{24 \pi m_{D^{*}}^{2}}\left|k_{\pi}\right|^{3}
$$

where, pion momentum, $k_{\pi}$ is,

$$
k_{\pi}=\sqrt{\frac{\left(m_{D^{*}}^{2}-m_{D}^{2}+m_{\pi}^{2}\right)^{2}}{\left(2 m_{D^{*}}\right)^{2}}-m_{\pi}^{2}} .
$$

In Eq. 11, coupling $g_{D^{*} D \pi}^{2}=12.5 \pm 1, m_{D^{*}}$ and $m_{D}$ denote the masses of vector and pseudoscalar meson, respectively. From Eq. 11, we observe that the values of decay width 
depend upon the masses of vector and pseudoscalar mesons, i.e., $D^{*}$ and $D$, respectively. Using vacuum values for the masses of $D$ mesons, the values of decay width $\Gamma\left(D^{*+} \longrightarrow\right.$ $D^{0} \pi^{+}$) is observed to be $32 \pm 5 \mathrm{keV}$ [43]. However, as we discussed in our present work, the charmed mesons get modified in the hadronic medium and this must lead to the medium modification of decay width of these mesons. For example, if we consider the in-medium masses of vector mesons from our present work and for pseudoscalar mesons we use the in-medium masses from Ref. [36] (in this reference the mass modifications of pseudoscalar $D$ mesons were calculated using the chiral $\mathrm{SU}(4)$ model in nuclear and strange hadronic medium), then in nuclear medium $\left(f_{s}=0\right)$, at baryon density, $\rho_{B}=\rho_{0}$, the values of decay width, $\Gamma\left(D^{*+} \longrightarrow D^{0} \pi^{+}\right)$, are observed to be $219 \mathrm{keV}$ and $31 \mathrm{keV}$ at asymmetry parameter $I=0$ and 0.5 , respectively. In strange medium $\left(f_{s}=0.5\right)$, the above values of decay width will change to 84 and $25 \mathrm{keV}$ at $I=0$ and 0.5 , respectively. We observe that the decay width of heavy mesons vary appreciably because of medium modification of heavy meson masses. In our future work we shall evaluate in detail the effects of medium modifications of masses and decay constants of heavy charmed and bottom mesons on the above mentioned experimental observables.

\section{SUMMARY}

In short, we computed the mass shift and shift in decay constants of vector and axialvector, charm and bottom mesons, in asymmetric hadronic matter, consisting of nucleon and hyperons, using phenomenological chiral model and QCD sum rules, at zero as well as at finite temperature. For this, first the quark and gluon condensates were calculated using chiral hadronic model and then, using these values of condensates as input in QCD sum rules, the in-medium properties of vector and axial-vector mesons were evaluated. We observed a negative (positive) shift in the masses and decay constants of vector (axial-vector) mesons. The magnitude of shift increases with increase in the density of baryonic density of matter. The properties of mesons are seen to be sensitive for the isospin asymmetry as well as strangeness fraction of the medium. The isospin asymmetry of the medium causes the mass-splitting between isospin doublets. The presence of hyperons in addition to nucleons cause more decrease (increase) in the masses and decay constant of vector (axial-vector) mesons. The finite temperature of the medium (at finite baryonic density) is observed 
to cause an increase in the masses and decay constants of heavy mesons. The observed effects on the masses and decay constants of heavy vector and axial-vector mesons may be reflected experimentally in the production ratio of open charm mesons as well as in their decay width. The negative mass shift of charmed vector mesons as observed in present calculations may cause the formation of bound states with the nuclei as well as the decay

of excited charmonium states to $D^{*} \bar{D}^{*}$ pairs causing charmonium suppression. The present work on the in-medium mesons properties may be helpful in understanding the experimental observables of CBM and PANDA experiments of FAIR project at GSI Germany.

\section{Acknowledgments}

The authors gratefully acknowledge the financial support from the Department of Science and Technology (DST), Government of India for research project under fast track scheme for young scientists (SR/FTP/PS-209/2012).

[1] http://www.lepp.cornell.edu/Research/EPP/CLEO/

[2] http://belle.kek.jp/

[3] http://www-public.slac.stanford.edu/babar/

[4] M. Monteno (for the NA50 collaboration), Nucl. Phys. Proc. Suppl. 92, 43 (2001).

[5] S.S. Adler (for the PHENIX collaboration),Phys. Rev. Lett. 98, 232301 (2007) .

[6] Constantin Loizides (for the ALICE collaboration), J. Phys. G: Nucl. Part. Phys. 28, 124040 (2011) .

[7] S.S. Adler (for the PHENIX collaboration),Phys. Rev. C 71, 034908 (2005).

[8] T. Matsui and H. Satz, Phys. Lett. B 178, 146 (1986).

[9] P. Braun-Munzinger, I. Heppe, J. Stachel, Phys. Lett. B 465, 15 (1999) .

[10] P. Braun-Munzinger and J. Stachel, Phys. Lett. B 490, 196 (2000).

[11] M. I. Gorenstein, A. P. Kostyuk, L. McLerran, H. Stocker and W. Greiner, J. Phys. G: Nucl. Part. Phys. 28, 2151 (2002).

[12] L. Grandchamp and R. Rapp, Phys. Lett. B 523, 60 (2001).

[13] A. Capella, E G Ferreiro and A B Kaidalov, Phys. Rev. Lett. 85, 208 (2000). 
[14] K. Tsushima, D.H. Lu, A.W. Thomas, K.Saito and R.H. Landau, Phys. Rev. C 59, 2824 (1999).

[15] L. Tolós, C. Garcia-Recio, J. Nieves, O. Romanets, L. L. Salcedo, Few-Body Syst 54, 923 (2013).

[16] L. Tolos, C. Garcia and J. Nieves, Phys. Rev. C 80, 065202 (2009).

[17] K. Tsushima and F.C. Khanna, Phys. Lett. B 552, 138 (2003).

[18] G. Krein, A. W. Thomas and K. Tsushima, Phys. Lett. B 697, 136 (2011).

[19] Arata Hayashigaki, Phys. Lett. B 487, 96 (2000).

[20] T. Hilger, R. Thomas, B. Kampfer, Phys. Rev. C 79, 025202 (2009).

[21] T. Hilger and B. Kampfer, Nucl. Phys. Proc. Suppl. 207, 277 (2010).

[22] S. Zoschoke, T. Hilger and B. Kampfer, Eur. Phys. J. A 47, 151 (2011).

[23] T. Buchheim, T. Hilger and B. Kampfer, J. Phys. Conf. Ser. 503, 012006 (2014).

[24] T. Buchheim, T. Hilger and B. Kampfer, Nucl. Part. Phys. Proc. 258, 213 (2015).

[25] T. Buchheim, T. Hilger and B. Kampfer, EPJ Web Conf. 81, 05007 (2014).

[26] T. Buchheim, T. Hilger and B. Kampfer, Phys. Rev. C 91, 015205 (2014).

[27] L. Tolos, J. Schaffner-Bielich and A. Mishra, Phys. Rev. C 70, 025203 (2004).

[28] L. Tolos, J. Schaffner-Bielich and H. Stocker, Phys. Lett. B 63, 85 (2006).

[29] J. Hoffmann and M.F.M Lutz, Nucl. Phys. A 763, 90 (2005).

[30] M.F.M Lutz and C. L. Kopra, Phys. Lett. B 633, 43 (2006).

[31] L. Tolos, A. Ramos and T. Mizutani, Phys. Rev. C 77, 015207 (2008).

[32] L. Tolos, R. Molina and E. Oset, Nucl. Phys. A 82, 249C (2009).

[33] O. Romanets et.al., Nucl. Phys. A 914, 488 (2013).

[34] E. Oset, Int. J. Mod. Phys. E 21, 1230011 (2012).

[35] L. Tolos, Int. J. Mod. Phys. E 22, 1330027 (2013).

[36] Arvind Kumar and Amruta Mishra, Eur. Phys, J. A 47, 164 (2011).

[37] D. Pathak and A. Mishra, Phys. Rev. C 91, 045206 (2015).

[38] D. Pathak and A. Mishra, Int. J. Mod. Phys. E 23, 0073 (2014).

[39] Zhi-Gang Wang, Tao Huang, Phys. Rev. C 84, 048201 (2011).

[40] Zhi-Gang Wang, Int. J. Mod. Phys. A 28, 1350049 (2013) .

[41] Arvind Kumar, Adv. in High Energy Physics 2014, 549726 (2014) .

[42] D. Ebert, R.N. Faustov and V.O Galkin, Mod. Phys. Lett. A 17, 803 (2002). 
[43] V. M. Belyaev, V. M. Braun, A. Khodjamirian and R. Ruckl, Phys. Rev. D 51, 6177 (1995).

[44] E. V. Shuryak, Nucl. Phys. B 198, 83 (1982).

[45] M. Neubert, Phys. Rev. D 45, 2451 (1992).

[46] E. Bagan, P. Ball, V. M. Braun and H. G. Dosch, Phys. Lett. B 278, 457 (1992).

[47] D. J. Broadhurst, Phys. Lett. B 101, 423 (1981).

[48] S. C. Generalis, J. Phys. G 16, 785 (1990).

[49] C. A. Dominguez and N. Paver, Phys. Lett. B 246, 493 (1990).

[50] P. Gelhausen, A. Khodjamirian, A.A. Pivovarov and D. Rosenthal, Phys. Rev. D 88, 014015, (2013).

[51] Zhi-Gang Wang, arXiv:1501.05093 [hep-ph].

[52] K. Azizi, N. Er, H. Sundu, Eur. Phys. J. C 74, 3021 (2014).

[53] E Veli Veliev et.al., J. Phys.: Conf. Ser. 347, 012034 (2012).

[54] P. Papazoglou, D. Zschiesche, S. Schramm, J. Schaffner - Bielich, H. Stocker and W. Greiner, Phys. Rev. C 59, 411 (1999).

[55] Arvind Kumar and Amruta Mishra, Phys, Rev. C 82, 045207 (2010).

[56] S. Zschocke, O.P. Pavlenko, B. Kampfer, Eur. Phys. J. A 15, 529 (2002).

[57] Y. Kwon, C. Sasaki, W. Weise, Phys. Rev. C 81, 065203 (2010).

[58] Arvind Kumar and Amruta Mishra, Phys, Rev. C 81, 065204 (2010).

[59] A. Mishra and S. Schramm, Phys. Rev. C 74, 064904 (2006), A. Mishra, S. Schramm and W. Greiner, Phys. Rev. C 78, 024901 (2008) and H. Stöcker, and W. Greiner, Phys. Rev. C 59, 411 (1999).

[60] Yuji Koike and Arata Hayashigaki, Prog. Theo. Phys. 98, 631 (1997).

[61] R. Thomas, T. Hilger, B. Kampfer, Nucl. Phys. A 795, 19 (2007).

[62] A. Khodjamirian, Ch. Klein, Th. Mannel and Y. -M. Wang, JHEP 09, 106 (2011).

[63] Damir Becirevic et.al. JHEP 02, 042 (2012).

[64] M. J. Baker et.al. JHEP 07, 032 (2014).

[65] Dae Sung Hwang and Gwang-Hee Kim, Phys. Rev. D 55, 69440 (1997).

[66] M. Lutz, S. Klimt and W. Weise, Nucl. Phys. A 452, 521 (1992).

[67] Thomas D. Cohen, R. J. Furnstahl and David K. Griegel, Phys. Rev. Lett. 67, 961 (1991).

[68] G.Q. Li, C.M. Ko, Phys. Lett. B 338, 118 (1994).

[69] WANG Ping, ZHANG Zong-Ye and YU You-Wen, Commun. Theor. Phys. 36, 71 (2001). 
[70] Delfino, A., Dey, J., Dey, M. et al., Phys. Lett. B 363, 17 (1995).

[71] Guo, H., J. Physics (London) G. 25, 1701 (1999).

[72] K. Saito, K. Tsushima, A. W. Thomas, Mod. Phys. Lett. A 13, 769 (1998).

[73] J. Sollfrank, U. Heinz, Z. Phys. C 65, 111 (1995).

[74] M. Oertel, M. Buballa, J. Wambach, Conference: C01-09-26.3, LYCEN-2001-88.

[75] Zhou Li-Juan, Zheng Bo, Zhong Hong-Wei and Ma Wei-Xing, Chinese Phys. C 39, 033101 (2015).

[76] Shotaro Imai, Hiroshi Toki, Wolfram Weise, Nucl. Phys. A 913, 71 (2013).

[77] T. Hilger, R. Schulze, B. Kampfer, J. Phys. G 37, 094054 (2010). 